\title{
A Bayesian Approach to Electrical Conductivity Relaxation and Isotope ExchangeSecondary Ion Mass Spectrometry
}

Joshua Blair

Follow this and additional works at: https://researchrepository.wvu.edu/etd

\section{Recommended Citation}

Blair, Joshua, "A Bayesian Approach to Electrical Conductivity Relaxation and Isotope ExchangeSecondary Ion Mass Spectrometry" (2014). Graduate Theses, Dissertations, and Problem Reports. 5220.

https://researchrepository.wvu.edu/etd/5220

This Thesis is protected by copyright and/or related rights. It has been brought to you by the The Research Repository @ WVU with permission from the rights-holder(s). You are free to use this Thesis in any way that is permitted by the copyright and related rights legislation that applies to your use. For other uses you must obtain permission from the rights-holder(s) directly, unless additional rights are indicated by a Creative Commons license in the record and/ or on the work itself. This Thesis has been accepted for inclusion in WVU Graduate Theses, Dissertations, and Problem Reports collection by an authorized administrator of The Research Repository @ WVU. For more information, please contact researchrepository@mail.wvu.edu. 
A Bayesian Approach to Electrical Conductivity Relaxation and Isotope Exchange/Secondary Ion Mass Spectrometry

\author{
Joshua Blair
}

Thesis submitted

To the College of Engineering

at West Virginia University

In partial fulfillment of the requirements for the degree of

\author{
Master of Science in \\ Mechanical Engineering \\ David Mebane, Ph.D., Chair \\ Ever Barbero, Ph.D. \\ Xingbo Liu, Ph.D
}

Department of Mechanical Engineering

Morgantown, West Virginia

2014

Keywords: Bayesian statistics, ECR, SIMS, uncertainty

quantification, data fitting, non linear

Copyright 2014 Joshua Blair 


\section{ABSTRACT \\ A Bayesian Approach to Electrical Conductivity Relaxation and Isotope Exchange/Secondary Ion Mass Spectrometry Joshua Blair}

In this work, data from isotope exchange - secondary ion mass spectroscopy (IE-SIMS) and electrical conductivity relaxation (ECR) experiments are analyzed using a purely Bayesian approach. The new technique allows quantification of the uncertainty associated with fitting two parameters (the surface exchange coefficient, $\mathrm{k}$, and the bulk diffusion coefficient, D) to a single reaction-diffusion model. The behavior and reliability of the technique is analyzed by considering an idealized data set, where the parameters of interest are pre-defined. The associated MCMC routine finds the parameter location in less than 5,000 samples, despite the starting point being 8 orders of magnitude away. Real experimental data from two experiments conducted on the same material at the same temperature and very similar partial pressures are analyzed and compared and yield vastly differing results from those obtained in the original studies. 


\section{Dedications}

I would like to dedicate this work to my family, partner, and research advisor. Without the unconditional love and support of my mother, father, and brother, I would not have had the confidence to pursue any college education in the first place. I also dedicate this work in part to my longtime partner, Ariel. She has remained by my side through all of the tribulation I have endured over the past two years, and I owe her everything. Last, but certainly not least, I dedicate this work to my research advisor. I cannot hope to ever repay him for his immense patience and his remarkable ability to get me to where I need to be. I certainly could not have completed this work without his guidance and willingness to support me at any cost. I will always be grateful for the opportunity to work with him, and all of the sacrifices he made on my behalf. 


\section{Acknowledgements:}

First of all, I would like to thank my committee, Dr. David Mebane, Dr. Xingbo Liu, and Dr. Ever Barbero for their willingness to work with me and provide feedback on such short notice. I would also like to thank Dr. Kirk Gerdes, Dr. Thomas Kalapos, Dr. Harry Finklea, Dr. Harry Abernathy, and the rest of the ionics team at NETL in Morgantown, WV, for this work wouldn't exist without their support. 


\section{Table of Contents:}

1: Introduction 1

2: Theory and Implementation 3

2.1 Bayesian Calibration 3

2.2 Models 5

2.3 Implementation 7

3: Results and Discussion 8

3.1 Behavior of Routine on Idealized Data 8

3.2 Isotope Exchange/Secondary Ion Mass Spectroscopy 13

3.3 Electrical Conductivity Relaxation 17

4: Conclusions 28 


\section{Introduction}

Mixed ionic-electronic conductors (MIEC's) have been used for many years in solid oxide fuel cells (SOFCs), batteries, sensors, as well as other devices [1]. As such, the associated transport properties of MIECs is of particular interest. Knowledge of transport properties in MIECs naturally allows for a better prediction of the performance and degradation of fuel cells, among other things. Determination of these properties is not a trivial matter, as no current experiment or technique is capable of measuring the properties directly. Two commonly used methods are electrical conductivity relaxation (ECR) and isotope-exchange secondary ion mass spectroscopy (IE-SIMS). Both techniques require fitting curves to experimental data and "selecting" the set of transport properties that yields the curve or curves that best fit the data. The transport properties of interest are the rate of exchange of ions across the surface of the MIEC $(k)$, and the rate of diffusion of ions into the bulk of the MIEC $(D)[2]$.

The IE-SIMS experiment is regarded as the most precise currently available and produces the lowest uncertainty with respect to transport properties. The nature of the experiment involves exposing a portion of a sample, such as one face of a rectangular parallel-piped to a certain partial pressure of oxygen for a sufficiently long time. The experiment then allows one to analyze the diffusion pattern as a function of distance from the penetrated surface. Commonly, the isotope ${ }^{18} \mathrm{O}$ is used due to its naturally low abundance. The diffusion of a rare isotope is much easier to keep track of than its more abundant counterpart. By knowing the rate of surface exchange and rate of bulk diffusion of the rare isotope, the total rates can be determined by direct proportionality. The transport properties are directly related to the diffusion profile via the reaction-diffusion model discussed below. The problem with the IE-SIMS experiment is that it requires very particular, expensive equipment that is difficult to operate $[3,4,5]$.

The ECR experiment provides a less expensive and more practical way to estimate transport properties of MIECs. The results from these experiments tend to contain more uncertainty than a SIMS experiment performed on the same given material. ECR involves inducing a small, rapid change in oxygen partial pressure, disturbing the equilibrium in the sample. The disturbance creates an electrostatic potential difference between the surface and bulk of the sample, which changes the electrical conductivity. By measuring the electrical conductivity as a function of the elapsed time from the step-change in the ambient partial pressure, the transport properties that best fit the data can then be chosen in the same way as in the SIMS experiment $[6,7]$. By knowing the value of the potential as a function of time and the sample dimensions, one can calculate the potential gradient as well as electrical conductivity as a function of time in the following way [8]:

$$
\begin{gathered}
J=E \sigma=\text { const. } \\
E=-\nabla \phi(x, t) \\
\nabla(\phi(x, t))=\frac{\delta(\phi)}{\delta x} \\
\sigma(t)=\frac{-J(x, t))}{\frac{\delta(\phi x}{\delta x}}
\end{gathered}
$$

There are two primary issues that could arise when fitting two or more parameters to a single model. The first issue is the so-called parameter identifiability. That is, a parameter is unidentifiable if changing it by a large amount produces a very small change in the model that the parameter belongs to. This issue arises when the model has neighborhoods of low sensitivity to changes in a particular fitted parameter $[2,9,10]$. The second issue has to do with the nature of the experiments. It is possible for a material to have a much slower surface reaction rate than bulk reaction rate, 
or vice versa. In the former case, the calculated bulk diffusion coefficient will be smaller than its actual value. It is also possible for surface incorporation and bulk diffusion to be co-limiting. A simple solution to this problem is to conduct one experiment for each parameter, where the physical dimensions of one sample is thin enough to disregard bulk diffusion, and the other sample large enough to ignore surface exchange. However, it is not clear in advance what an adequate sample size would be in either case to be able to ignore one parameter. For this reason, both parameters will be fit to a single model in this work.

The best way to fit multiple parameters to a single model is a widely discussed topic. Some gradient methods require the initial guess of parameters to be close to the actual value. Other methods rely on linearizing the non-linear least squares cost function, despite the fact that it has been shown that the non-linear behavior of this function can be important in some areas, and other methods require a great deal of the results ahead of time [11]. A Bayesian approach was described and applied to ideal, SIMS and ECR data. It will be shown that this technique does not require one to know anything about the value of the parameters or their uncertainty ahead of time, and it will be shown that the "initial guess" can be nearly any distance away from the actual parameter value and this technique will still find the optimal parameters.

Routines were written in MATLAB to use this technique. One was created for ECR, and one for SIMS. The only differences are sample geometry, and that one accepts ECR data and the other accepts SIMS data. The nature of the routine and the calculations performed with the data throughout each routine is identical. In particular, a Markov Chain Monte Carlo routine was used to analyze the data, and the nature of the routine will be explained below $[12,13,14]$.

\section{Theory and Implementation}

\subsection{Bayesian Calibration}

The mathematics associated with the Bayesian calibration will now be described. Start from the well-known theorem from probability theory:

$$
P(A \mid B)=P(A \bigcap B) / P(B),
$$

and

$$
P(B \mid A)=P(B \bigcap A) / P(A)=P(A \bigcap B) / P(A)
$$

After solving for $P(A \bigcap B)$ in eq. (2) we obtain,

$$
P(A \mid B)=\frac{P(B \mid A) P(A)}{P(B)} .
$$

Eq. (3) is known as Bayes Theorem and holds for any events A and B. In the context of this technique, we are interested in evaluating $P(\theta \mid z)$, where $\theta$ is the vector of fitted parameters of a particular experiment, and $z$ is the vector of experimentally obtained data.

This mathematical structure assumes the existence of a joint probability distribution between $\theta$ and $z$, so that

$$
P(\theta \mid z)=\frac{P(z \mid \theta) P(\theta)}{P(z)}
$$


Due to the existence of the joint distribution between the parameters of $\theta$ and the data $z$, the denominator of eq. (4) must be obtained through summation of the continuous variable $\theta$, that is

$$
P(z)=\int_{\theta} P(z \mid \theta) P(\theta)
$$

The integral in eq. (5) is unknown, but it should be noted that $\mathrm{P}(\mathrm{z})$ is a constant since the data is fixed. Since $\mathrm{P}(\mathrm{z})$ does not depend on $\theta$, the statement below can be made.

$$
P(\theta \mid z) \propto \mathcal{L}(z \mid \theta) P(\theta) .
$$

Here, $P(z \mid \theta)$ is called the likelihood of $z$ given $\theta$ and is a number proportional to the probability that $\theta$ is a realization of the data $z \cdot \mathrm{P}(\theta)$ is called the prior distribution. This technique starts by sampling from the prior. $P(\theta \mid z)$ is called the posterior distribution, and will serve as the set of results from the analysis.

By using a uniform prior, and specifying parameter bounds, statistical bias can be eliminated at the level of the prior [15]. It is then only necessary to ensure that the choice of bounds contain all possible parameter values. It should also be noted that in the uniform prior case, $P\left(\theta_{i}\right)=P\left(\theta_{j}\right)$ for any choice of $\mathrm{i}$ and $\mathrm{j}$, so $P(\theta)$ can be "absorbed" into the proportionality constant. That is, we can say that

$$
P(\theta \mid z) \propto \mathcal{L}(z \mid \theta)
$$

The value $\mathcal{L}(z \mid \theta)$ has no meaning by itself. In order to give significance to the likelihood, consider two vectors $\theta_{i}$ and $\theta_{j}$. By eq. (7), $P\left(\theta_{i} \mid z\right)=\alpha \mathcal{L}\left(z \mid \theta_{i}\right)$, and $P\left(\theta_{j} \mid z\right)=\alpha \mathcal{L}\left(z \mid \theta_{j}\right)$. By direct substitution, it is seen that

$$
\frac{P\left(\theta_{i} \mid z\right)}{P\left(\theta_{j} \mid z\right)}=\frac{\mathcal{L}\left(z \mid \theta_{i}\right)}{\mathcal{L}\left(z \mid \theta_{j}\right)}
$$

From the above equation, it is clear that the likelihood function has meaning when two sets of parameters are being compared to one another. By comparing the value of the likelihood function between sets of parameters, we can then draw conclusions about the relative value of the probability distribution function between those parameters. This idea of likelihood ratios is the central idea used in this analysis [12].

By examining the form of the likelihood in equation 10 below, it is seen that the form is a product of Gaussian distributions. It is assumed from the outset that there exists a Gaussian distribution at every data point, centered at the particular data point. Clearly, curves that are intended to fit the data will not fit the data perfectly. Generally, at every data point, the difference $z_{i}-y_{i}$, where $z_{i}$ and $y_{i}$ are the $i^{\text {th }}$ data point and corresponding $i^{\text {th }}$ prediction from a particular draw of parameters, is non-zero. Also, it is expected that the value of $z_{i}-y_{i}$ will vary as $i$ varies, so that after a large number of samples have been recorded, each data point will have its own unique Gaussian distribution with its own unique variance. Consideration also must be given to the correlation between points. The correlation can be easily visualized by considering if the $j^{\text {th }}$ prediction value will be affected by changing the value of the $i^{\text {th }}$ data point. It is clear that large changes in the $i^{\text {th }}$ data point will have some effect on the $j^{\text {th }}$ prediction value. So, in general, a covariance matrix $\Sigma$ is needed to fully describe the distribution of the error.

$$
z=Y(\theta)+\Sigma(\psi)
$$


In this work, it is assumed and known that the model used to fit the data is a reliable one, and the precision of both ECR and SIMS experiments is high enough so that the large changes mentioned above are not an issue. It is safe then to ignore any correlations that may be present, as their effect is considered negligible. The complicated covariance matrix reduces to a single number called $\psi$. The error, therefore, is characterized by a single Gaussian distribution with variance $\psi$. Here, $\Sigma=\operatorname{cov}\left(x_{i}, x_{j}\right)$ is the covariance matrix representing the correlation of the error between points $x_{i}$ and $x_{j}$.

$$
z=Y(\theta)+\epsilon(\psi)
$$

where $Y$ is the model pertaining to the experiment, and $\epsilon$ is Gaussian white noise with variance $\psi$. Given a dataset $z$, the difference between the model and dataset, $\epsilon=z-Y(\theta)$ is normally distributed, with mean zero and covariance equal to the identity times $\psi$ :

$$
\mathcal{L}(\theta \mid z)=\frac{1}{(2 \pi)^{N / 2} \psi^{N / 2}} \exp \left[-\frac{(z-Y(\theta))^{T}(z-Y(\theta))}{2 \psi}\right]
$$

In principle, the error $\psi$ could take on a single value, but it is not known in advance what its value is, therefore $\psi$ will be sampled alongside $k$ and $D$, and a distribution will be obtained for $\psi$. Equations (4-5) are then rewritten as:

$$
P(\theta, \psi \mid B)=\mathcal{L}(B \mid \theta, \psi) p(\theta) p(\psi)\left[\int \mathcal{L}(B \mid \theta, \psi) p(\theta) p(\psi) d \theta d \psi\right]^{-1}
$$

and the posterior is now a joint distribution over $\theta$ and $\psi$. Integration over $\psi$ then recovers a distribution joint over just the physical parameters $k^{*}$ and $D^{*}$.

For $\psi$, it is computationally advantageous to use an inverse gamma prior, since the inverse gamma distribution is "conjugate" to the likelihood for fixed $\theta$, meaning that there is an analytical solution for the conditional posterior distribution given $\theta$. The inverse gamma prior contains all of the requirements for the variance $\psi$. Namely, it is bounded from below at zero, and the IG is still considered a vague prior because, like the uniform prior, it can be made to be as broad as needed $[12]$.

\subsection{Models}

The models used in both the ECR and SIMS experiments are very similar to one another. First the SIMS model will be discussed. Consider a semi-infinite slab of material with initial uniform concentration of solute $C_{2}$ placed in an environment with ambient concentration $C_{0}$, and the solute concentration as a function of $x$ and time is governed by the one-dimensional diffusion equation:

$$
\frac{\partial C}{\partial t}=D \frac{\partial^{2} C}{\partial x^{2}}
$$

where $D$ is the bulk diffusion coefficient, and $C$ is the concentration of a diffusing substance as a function of depth and time.

To obtain the desired two-step process, the surface must be considered. Let $C_{s}$ be the concentration at the interface of the material and surface. The assumption will be made that the 
rate of surface exchange is directly proportional to the concentration difference between the surface and environment. This assumption produces the following boundary condition involving the concentration gradient at the surface:

$$
D \frac{\partial C}{\partial x}=k\left(C_{s}-C_{0}\right)
$$

The initial condition is:

$$
C(x, 0)=C_{2}
$$

The solution to equation (12) with conditions (13) and (14) is:

$$
C(x, t)=C_{2}+\left(C_{0}-C_{2}\right)\left[\operatorname{erfc}\left(\frac{x}{2 \sqrt{D^{*} t}}\right)-\exp \left(l x+l^{2} D^{*} t\right) \operatorname{erfc}\left(\frac{x}{2 \sqrt{D^{*} t}}+l \sqrt{D^{*} t}\right)\right]
$$

Some simple algebra leads to the normalized equation:

$$
\frac{C(x, t)-C_{2}}{C_{0}-C_{2}}=\operatorname{erfc}\left(\frac{x}{2 \sqrt{D^{*} t}}\right)-\exp \left(l x+l^{2} D^{*} t\right) \operatorname{erfc}\left(\frac{x}{2 \sqrt{D^{*} t}}+l \sqrt{D^{*} t}\right)
$$

where $l=k^{*} / D^{*}, c(x)$ is the isotope fraction at a distance $x$ away from the surface, $c_{2}$ is the isotope fraction in the enriched gas, and $c_{0}$ is the background isotope fraction.

In the context of SIMS, $C$ refers to the concentration of solute, ${ }^{18} O$, in the sample. This oxygen concentration is measured directly using specialized equipment.

The ECR is set up very similarly, but the material is a plane sheet with a definite size as opposed to the semi-infinite slab from SIMS. Now consider a one-dimensional material with $-l_{x}<x<l_{x}$ subject to the same boundary conditions as in the SIMS case. The solution to this problem is:

$$
\frac{C(t)-C_{2}}{C_{0}-C_{2}}=1-\sum_{i=1}^{\infty} \frac{2 l_{1} \exp \left(-\beta_{1 i}^{2} D^{*} t / x_{1}^{2}\right)}{\beta_{1 i}^{2}\left(\beta_{1 i}^{2}+l_{1}^{2}+l_{2}\right)}
$$

where $x_{p}$ is the half-width of the sample in dimension $p, l_{p}=x_{p} k^{*} / D^{*}$, and $\beta_{p q}$ is the $q^{\text {th }}$ solution to the equation $\beta_{p q} \tan \beta_{p q}=l_{p}$. For ECR, C refers to the concentration of oxygen nonstoichiometry[16]. It is then assumed that the changes in partial pressure are sufficiently small so that a linear relationship can be assumed between the $\mathrm{pO}_{2}$ change and the conductivity change. That is to say:

$$
\frac{C(t)-C_{2}}{C_{0}-C_{2}}=\frac{\sigma(t)-\sigma_{2}}{\sigma_{0}-\sigma_{2}}
$$

where $\sigma(t)$ is the measured conductivity at time $t, \sigma_{0}$ is the conductivity before the $\mathrm{pO}_{2}$ change, and $\sigma_{\infty}$ is the conductivity at an infinite time after the $\mathrm{pO}_{2}$ change. Equation (17) must be "expanded" into three dimensions to describe the solution for a rectangular parallel piped geometry in the following way[6]:

$$
\begin{array}{r}
\frac{\sigma(t)-\sigma_{\infty}}{\sigma_{0}-\sigma_{\infty}}=1-\sum_{i=1}^{\infty} \sum_{j=1}^{\infty} \sum_{k=1}^{\infty} \frac{2 l_{1} \exp \left(-\beta_{1 i}^{2} D^{*} t / x_{1}^{2}\right)}{\beta_{1 i}^{2}\left(\beta_{1 i}^{2}+l_{1}^{2}+l_{2}\right)} \frac{2 l_{2} \exp \left(-\beta_{2 j}^{2} D^{*} t / x_{2}^{2}\right)}{\beta_{2 j}^{2}\left(\beta_{2 j}^{2}+l_{2}^{2}+l_{2}\right)} \\
\frac{2 l_{3} \exp \left(-\beta_{3 k}^{2} D^{*} t / x_{3}^{2}\right)}{\beta_{3 k}^{2}\left(\beta_{3 k}^{2}+l_{3}^{2}+l_{3}\right)}
\end{array}
$$


When the ambient partial pressure changes, it causes the system to come out of equilibrium. Reequilibration immediately ensues. First, the oxygen vacancy concentration at the surface changes to accommodate for the new partial pressure in the environment. The change in the surface concentration causes a gradient in the electrostatic scalar potential across the sample. A circuit with constant current (and therefore constant current density) is setup to measure this potential $[6,7]$.

\subsection{Implementation}

Since there is no explicit way to write down the form of the posterior distribution, a numerical method to obtain the posterior is employed. The use of the likelihood ratios of equation (8) together with the so-called Metropolis criterion form the heart of the routine. This type of routine is known as a Markov Chain Monte Carlo sampling system [17]. A Markov chain is a process in which the next draw depends only upon the current draw. That is $\theta_{i}=f\left(\theta_{i-1}\right)$ only. A Monte Carlo sampling process is simply one that involves a large number of random samples and more weight is placed in regions of higher probability than those of low probability. One advantage to MCMC sampling is the guarantee of convergence in the limit of infinite samples to the correct distribution. It will be shown, however, that in the context of SIMS and ECR that heart of the distribution (neighborhood of the true $k$ and $D$ ) is located in no more than about 3000 samples, even when the starting point is several orders of magnitude from the true neighborhood, and the important statistics of the posterior distribution are observed with about 10,000 samples.

The MCMC sampling proceeds stepwise from an initial user-selected draw $\left\{\theta_{0}, \psi_{0}\right\}$, from the uniform prior distribution, with new draws proposed and accepted or rejected in sequence. The acceptance and rejection criteria are different for $\theta$ and $\psi$. Given an accepted draw $\theta_{i-1}$, a new proposal $\hat{\theta}_{i}$ is accepted or rejected according to the Metropolis criterion:

$$
\begin{aligned}
& \mathcal{L}\left(\hat{\theta}_{i}\right) \geq \mathcal{L}\left(\theta_{i-1}\right) \rightarrow \theta_{i}=\hat{\theta}_{i} \\
& \mathcal{L}\left(\hat{\theta}_{i}\right)<\mathcal{L}\left(\theta_{i-1}\right) \rightarrow\left\{\begin{array}{l}
P\left(\theta_{i}=\hat{\theta}_{i}\right)=\mathcal{L}\left(\hat{\theta}_{i}\right) / \mathcal{L}\left(\theta_{i-1}\right) \\
P\left(\theta_{i}=\theta_{i-1}\right)=1-\mathcal{L}\left(\hat{\theta}_{i}\right) / \mathcal{L}\left(\theta_{i-1}\right)
\end{array}\right.
\end{aligned}
$$

Notice that equation (22) implies from equation (8) that $P\left(\theta_{i}\right)>P\left(\theta_{i-1}\right)$.

For $\psi$, the conjugate inverse gamma prior leads to a sampling regime wherein new proposals will always be accepted for a given $\theta$ (Gibbs sampling). Using the notation

$$
I G(\nu, \tau)=\frac{\tau^{\nu}}{\Gamma(\nu)} \psi^{-\nu-1} \exp \left(\frac{-\tau}{\psi}\right)
$$

if the prior distribution for $\psi$ is $I G(\nu, \tau)$, then the conditional posterior given $\theta$ is $I G\left(\nu^{\prime}, \tau^{\prime}\right)$, where

$$
\begin{gathered}
\nu^{\prime}=\nu+N / 2 \\
\tau^{\prime}=\tau+(B-Y(\theta))^{T}(B-Y(\theta)) / 2
\end{gathered}
$$

Given $\theta, \psi$ can simply be drawn from this known posterior distirbution. Note that neither of these criteria require explicit evaluation of the integral found on the right-hand side of equation (11).

Consider an accepted draw, $\theta_{i}$, the next proposed draw $\hat{\theta}_{i+1}$ is determined by placing it on a normal distribution centered at $\theta_{i}$ with variance $\varsigma$, so that the probability of $\hat{\theta}_{i+1}$ being permitted 
to metropolis increases as $\hat{\theta}_{i+1}$ approaches $\theta_{i}$, and the average step size from $\theta_{i}$ to $\hat{\theta}_{i+1}$ is determined by $\varsigma$. It is desired to compare points that are as near to each other as possible. The step size must be large enough that over the course of a few thousand samples, the routine can cover several orders of magnitude. $\varsigma_{D}, \varsigma_{k}$, and determines the step size, on average, of each new proposal. These values are on a common-log scale. For example, choosing $\varsigma_{D}=0.1$ will result in successive proposals for D being approximately 1/10th of an order of magnitude apart.

Sampling is performed one parameter at a time. Consider an accepted ordered pair $\left(k_{0}, D_{0}\right)$. First, $k_{1}$ is sampled from the prior, then its value is placed on a Gaussian distribution with variance $\varsigma$. Let $G\left(k_{0}, \varsigma_{k}, k_{1}\right)$ represent the value of the Gaussian distribution with mean $k_{0}$, variance $\varsigma_{k}$ at $k_{1}$. If $G\left(k_{0}, \varsigma_{k}, k_{1}\right)>R(0,1)$, where $R(0,1)$ is a random number in the open interval $(0,1)$, then $k_{1}$ is permitted to metropolis. Otherwise, a new $k_{1}$ is drawn from the prior. Then, the metropolis step compares $\mathcal{L}\left(k_{1}, D_{0}\right)$ with $\mathcal{L}\left(k_{0}, D_{0}\right)$, as described above. Once metropolis accepts $k_{1}$, the entire process is then repeated by first drawing $D_{1}$ from the prior. When the process for $D_{1}$ occurs, metropolis will compare $\mathcal{L}\left(k_{0}, D_{1}\right)$ with $\mathcal{L}\left(k_{0}, D_{0}\right)$, so that each parameter is sampled seperately.

Equation (20) forces consecutive accepted draws to march in the direction of higher probability. Equation (21) gives the routine the opportunity to "escape" a local maximum neighborhood and march toward a maximum located elsewhere in the parameter space. It should be noted that while equations (20-21) usually suffice to allow the routine to find the neighborhood of the global maximum likelihood, the equations by themselves do not guarantee to find the global max with a finite number of samples. However, with the use of graphics, it is easy to see what regions of the parameter space have been explored and what regions have not. To explore a previously unexplored region, one must simply start the routine in the region of interest, and ensure that $\varsigma$ is sufficiently small. When the global max neighborhood is found, the routine will continue to draw from that neighborhood and begin to reveal the details of the distribution. After a sufficient number of samples is taken, contour plots can be inspected to reveal the relative density of certain neighborhoods of the posterior. Also, one can get an idea of parameter identifiability by looking at the contour plots; a poorly identifiable parameter will have its high-density region elongated heavily along the axis of the parameter. After the routine finishes, a batch-means test is performed on the posteriors to assess convergence as follows [18]:

$$
\varphi_{B M}=\frac{b}{a-1} \sum_{j=1}^{a}\left(\bar{\theta}_{j}-\bar{\theta}\right)^{2}
$$

where $\bar{\theta}_{j}$ is the mean for batch $j$ and $\bar{\theta}$ is the overall sample mean. The simulation has then converged to the above-defined standard when

$$
\mathcal{T} \varphi_{B M} / \sqrt{M} \leq \delta^{2}
$$

where $\mathcal{T}$ is the Student's T-distribution quantile corresponding to $95 \%$ confidence and $a-1$ degrees of freedom, and $\delta$ is a standard deviation corresponding to $1 \%$ of the overall mean, $M$ is the number of samples, $a$ is the number of samples per batch, and $b$ is the number of batches. 


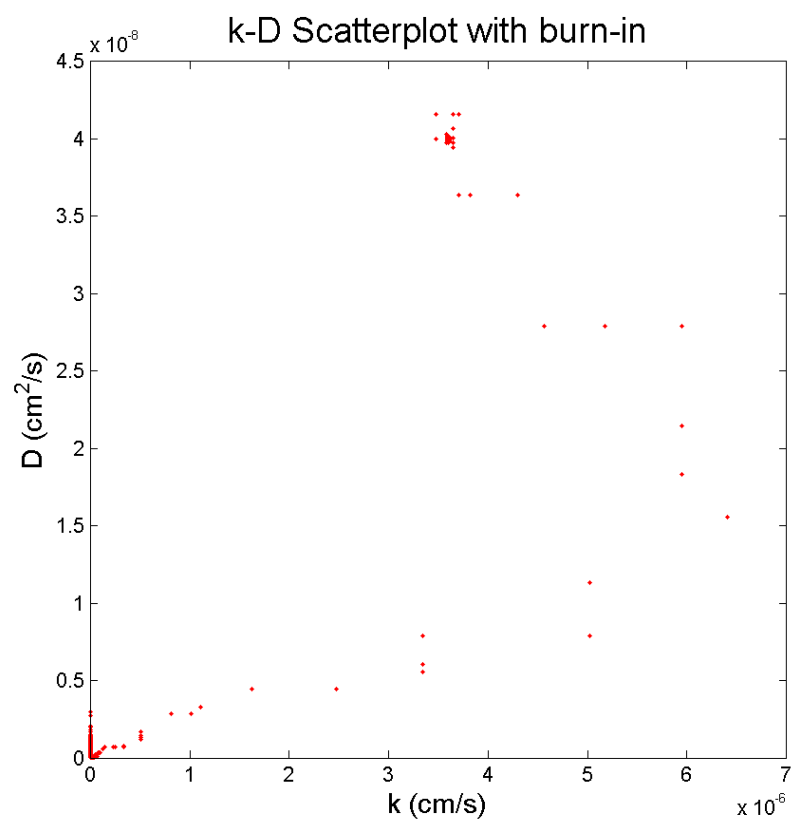

Figure 1: Burn in plot for ideal data. The starting point was in the lower left corner at $\left(k_{0}, D_{0}\right)=$ $\left(10^{-15}, 10^{-15}\right)$.

\section{Results and Discussion}

\subsection{Behavior of routine on idealized data}

In order to obtain preliminary information on the way the routine behaves, a set of ideal data was produced and the simulation was performed using this data. A small amount of noise was added to ensure that the value of the likelihood remained a real number. The bounds on the prior for both parameters were arbitrarily set to $10^{-15}$ and $10^{-1}$ for the minimum and maximum, respectively. The values of $\mathrm{k}$ and $\mathrm{D}$ were pre-chosen to be $k=3.6 \times 10^{-6}$, and $D=4 \times 10^{-8}$, respectively. The purpose of this is three-fold. The first reason for the ideal data is to see if the routine can find the region containing the optimum $(k, D)$ ordered pair in the first place. The second reason, provided the optimum is found, is to see if the routine can locate the optimum if the initial guess is far from the optimum. The third reason is to see how the routine behaves when changing the value of $\varsigma$.

Figure 1 shows the burn in pattern from starting the simulation at the prior minimum. That is, $\left(k_{0}, D_{0}\right)=\left(1 \times 10^{-15}, 1 \times 10^{-15}\right)$. Figures 2 through 4 are just zoomed in views of the burn in pattern. The heart of the distribution can be clearly seen in figure 1. Figure 5 shows the burn in pattern for starting the simulation near the prior maximum. The starting point can be seen as the right-most point in figure 5. The simulation then marches to the left, then up, and back down, where the density begins to increase. The heart of the distribution is finally located and is in the bottom left corner of figure 5 . Figure 6 is the result of removing the burn-in iterations from figures 1 through 5 . The simulation converged to the same distribution within the first 5,000 iterations, despite the fact that one run started at the prior maximum and the other started at the prior minimum. Finally, figures $7, ' 8$, and ' 9 show the effect of changing $\varsigma$. Setting $\varsigma=1$ means 


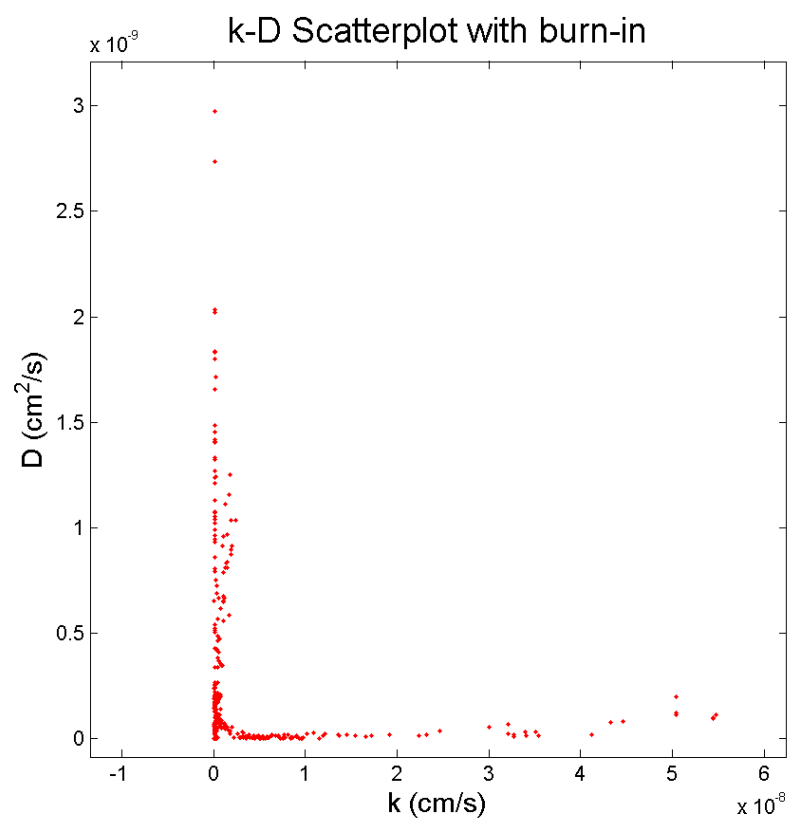

Figure 2: Zoomed in verison of 1 . The effect of $\varsigma_{D}=0.1$ is clearly visible by looking at the large gap in the upper left hand corner.

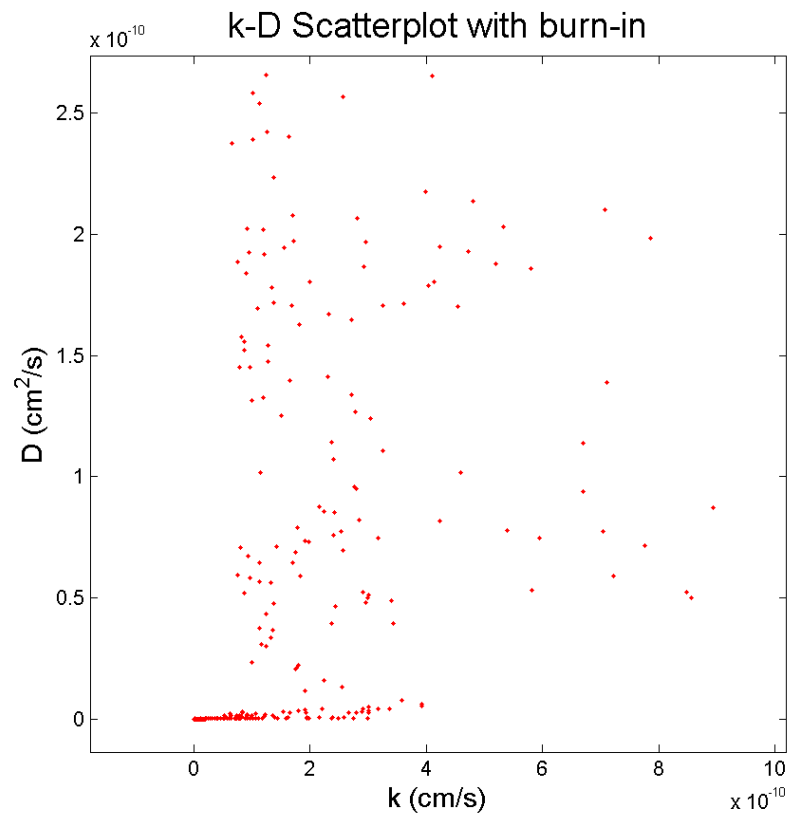

Figure 3: After zooming in further, the logarithmic nature of the proposals becomes clear when comparing the relative density of points in the lower left corner with the density elsewhere. 


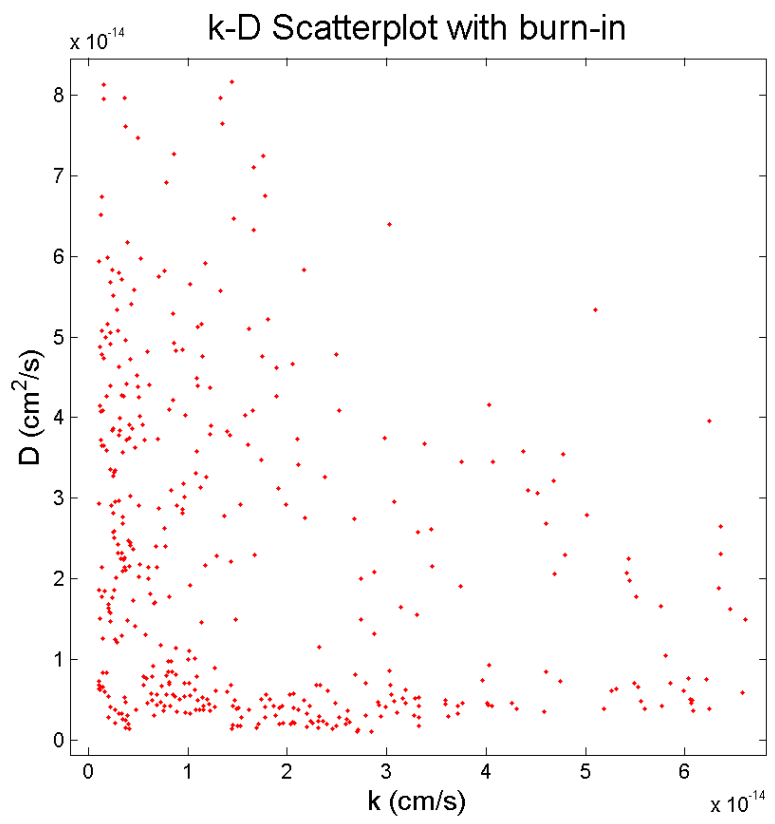

Figure 4: Figure 1 zoomed all the way in to see the starting point.

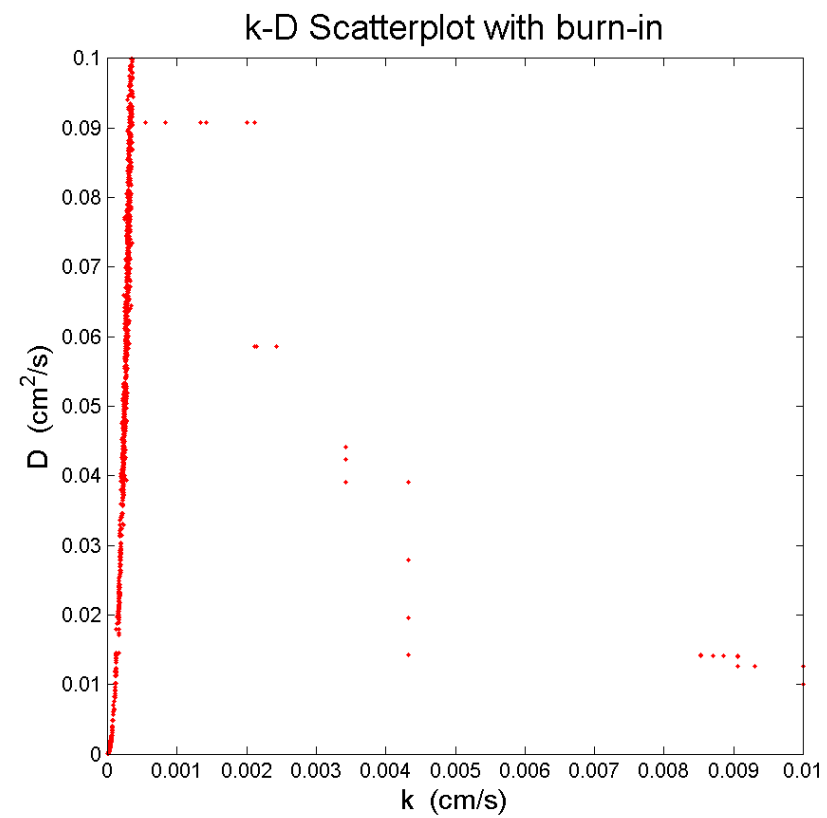

Figure 5: Burn-in for ideal data from choosing starting value near prior maximum.

that there is roughly an order of magnitude between successive proposals. As figure 8 shows, the distribution has not converged at all. Therefore, it is not recommended to use this value of $\varsigma$ in this situation. Figure 9 shows a nice distribution that has clearly converged, and plainly displays the 


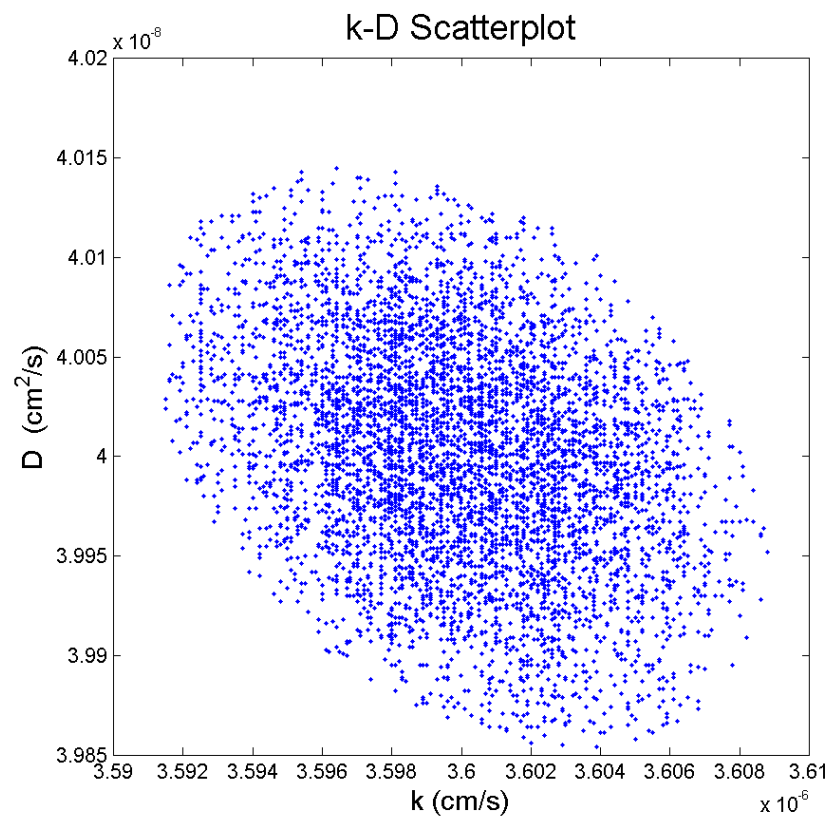

Figure 6: Scatter plot after removing burn-in from fig. 1 and 5.

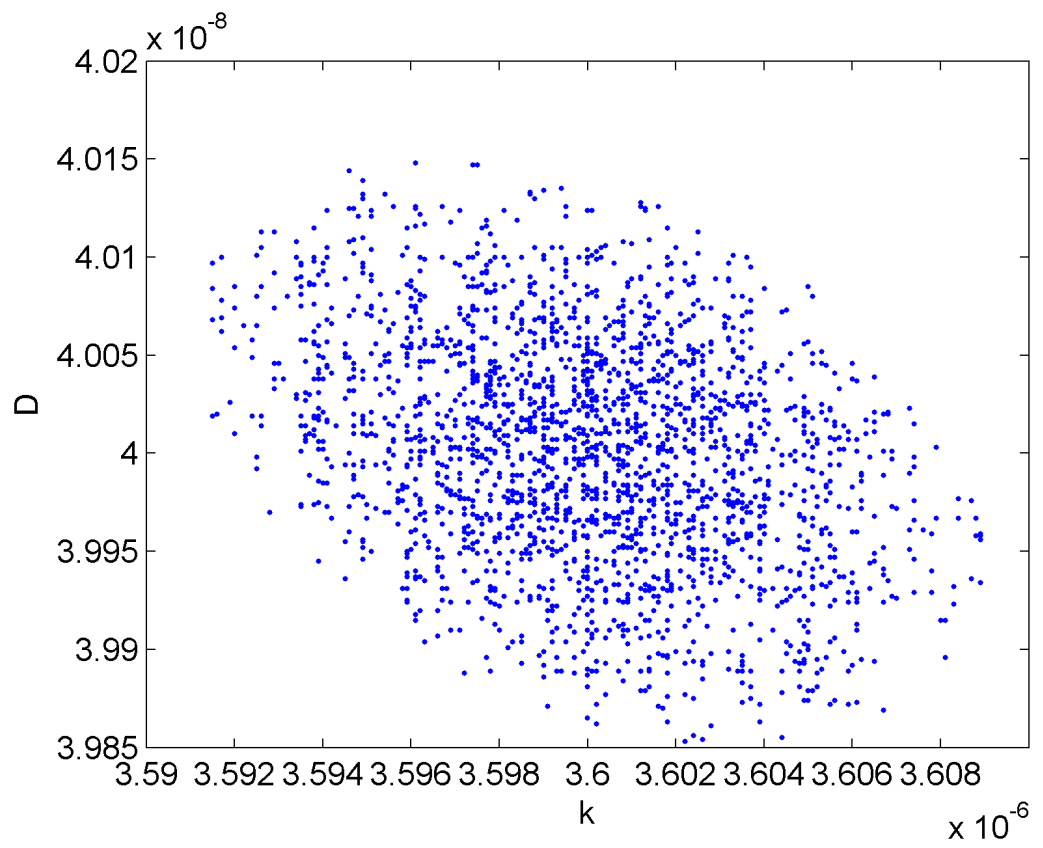

Figure 7: $\quad$ Scatter plot for $\varsigma_{i}=0.1$

relative density of points in the distribution. However, to obtain the results, the starting point had to be made closer to the optimum. So setting $\varsigma=0.01$ is not recommended for locating the heart of the distribution, but given the heart of the distribution, this value of $\varsigma$ can be used to resolve 


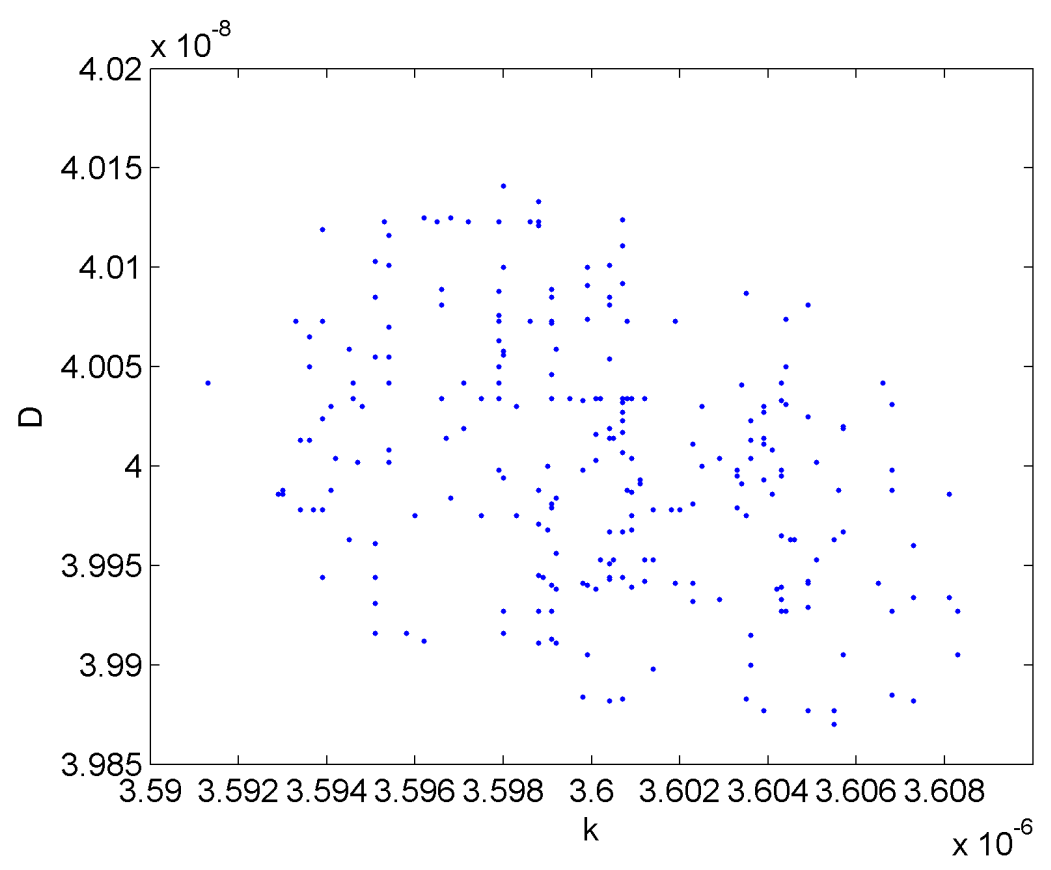

Figure 8: Scatter plot for $\varsigma_{i}=1$.

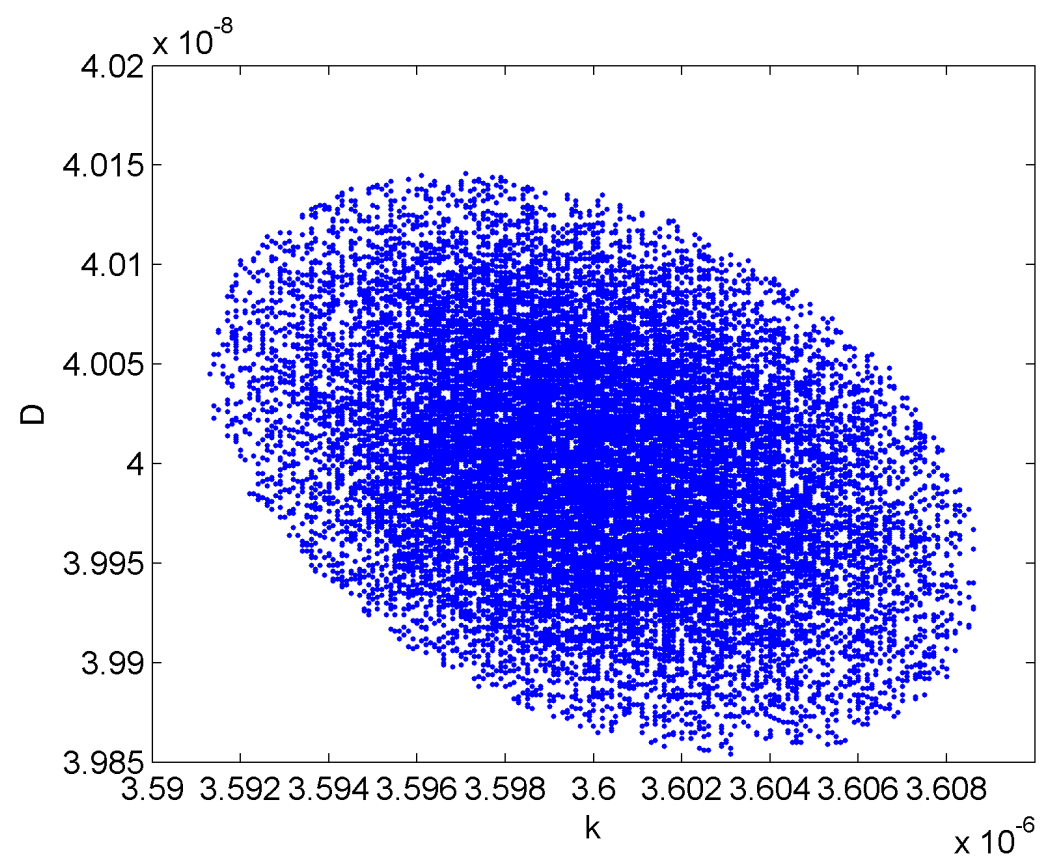

Figure 9: Scatter plot for $\varsigma_{i}=0.01$ for ideal data.

the details. It seems that $\varsigma=0.1$ is the sweet spot. Using this value allows the routine to quickly navigate the parameter space without there being too much space between proposals. Also, once the heart is found, the routine will stay there, allowing the simulation to converge and begin to 
map out the details of the distribution.

\subsection{Isotope Exchange / Secondary Ion Mass Spectroscopy}

Experimental data was drawn from Pietrowski [19]. In their paper, the effect of a wet exchange versus a dry exchange was analyzed. For the dry exchange, an ${ }^{18} \mathrm{O}$ gas was used, and for the wet exchange, $\mathrm{H}_{2}^{18} \mathrm{O}$ water vapor was used. The major findings were that $\mathrm{D}$ does not vary whether a wet or dry exchange occurs, but $\mathrm{k}$ increases by over an order of magnitude when a wet exchange is used. The data analyzed pertains to the wet exchange of oxygen in YSZ originally presented in Ref. [19], for the rough case. Results for the diffusion coefficients and surface exchange coefficients are presented in the table below in the context of 95 percent confidence intervals, along with the corresponding distribution modes. The intervals in all cases were calulated by removing the parameters mapped to the lowest likelihood values, such that the parameters corresponding to the 95 percent highest likelihood values remain. All of the figures below are produced automatically when the routine finishes. Figures from one particular data set are included here, but the same type of figures were produced and used for all data sets.

Table 1: 95 percent confidence intervals for the SIMS analysis.

\begin{tabular}{|l|l|l|c|r|}
\hline$p \mathrm{H}_{2} \mathrm{O}(\mathrm{mbar})$ & $k_{\min }$ & $k_{\max }$ & $D_{\min }$ & $D_{\max }$ \\
\hline 220 & $3.5949 \times 10^{-6}$ & $3.6829 \times 10^{-6}$ & $3.9567 \times 10^{-8}$ & $4.1040 \times 10^{-8}$ \\
\hline 125 & $2.5437 \times 10^{-6}$ & $2.5748 \times 10^{-6}$ & $4.8641 \times 10^{-8}$ & $4.9726 \times 10^{-8}$ \\
\hline 70 & $2.5346 \times 10^{-6}$ & $2.5727 \times 10^{-6}$ & $5.3324 \times 10^{-8}$ & $5.4754 \times 10^{-8}$ \\
\hline 18 & $9.7016 \times 10^{-7}$ & $9.8342 \times 10^{-7}$ & $3.4310 \times 10^{-8}$ & $3.5566 \times 10^{-8}$ \\
\hline 7 & $1.5661 \times 10^{-6}$ & $1.5839 \times 10^{-6}$ & $4.4068 \times 10^{-8}$ & $4.5266 \times 10^{-8}$ \\
\hline 2 & $1.6731 \times 10^{-7}$ & $1.7662 \times 10^{-7}$ & $3.9205 \times 10^{-8}$ & $4.6741 \times 10^{-8}$ \\
\hline
\end{tabular}

Table 2: Parameter modes for the SIMS analysis.

\begin{tabular}{|l|l|l|}
\hline $\mathrm{pH}_{2} \mathrm{O}(\mathrm{mbar})$ & $k_{\text {mode }}$ & $D_{\text {mode }}$ \\
\hline 220 & $3.650 \times 10^{-6}$ & $4.009 \times 10^{-8}$ \\
\hline 125 & $2.560 \times 10^{-6}$ & $4.910 \times 10^{-8}$ \\
\hline 70 & $2.560 \times 10^{-6}$ & $5.400 \times 10^{-8}$ \\
\hline 18 & $9.770 \times 10^{-7}$ & $3.490 \times 10^{-8}$ \\
\hline 7 & $1.580 \times 10^{-6}$ & $4.460 \times 10^{-8}$ \\
\hline 2 & $1.720 \times 10^{-7}$ & $4.170 \times 10^{-8}$ \\
\hline
\end{tabular}

Figure 10 shows all 500,000 sample iterations. The simulation starts at the right-most point and "marches" to the lower left corner, where the simulation reamins. In this case, it took only about fifty iterations for the simulation to find the heart of the distribution. After removing the burn-in iterations, figure 11 is obtained. Figure 11 is the heart of the distribution, and shows the span of the uncertainty. Due to the multitude of points, figure 11 does not provide a good sense of how dense the posterior is in a given region. Figures 12 and 13 are alternative representations of figure 11. The z-axis of figure 12 shows the density of points in various regions of the posterior space. The density in figure 12 is directly mapped into the colors of figure 13 . That is, the highest regions of figure 12 are mapped into the dark red region of figure 13, while the lowest non-zero regions of figure 12 are mapped into the blue regions of figure 13. The combination of figures 12 and 13 provide a superior alternative to standard error bars, since standard error bars provide no 


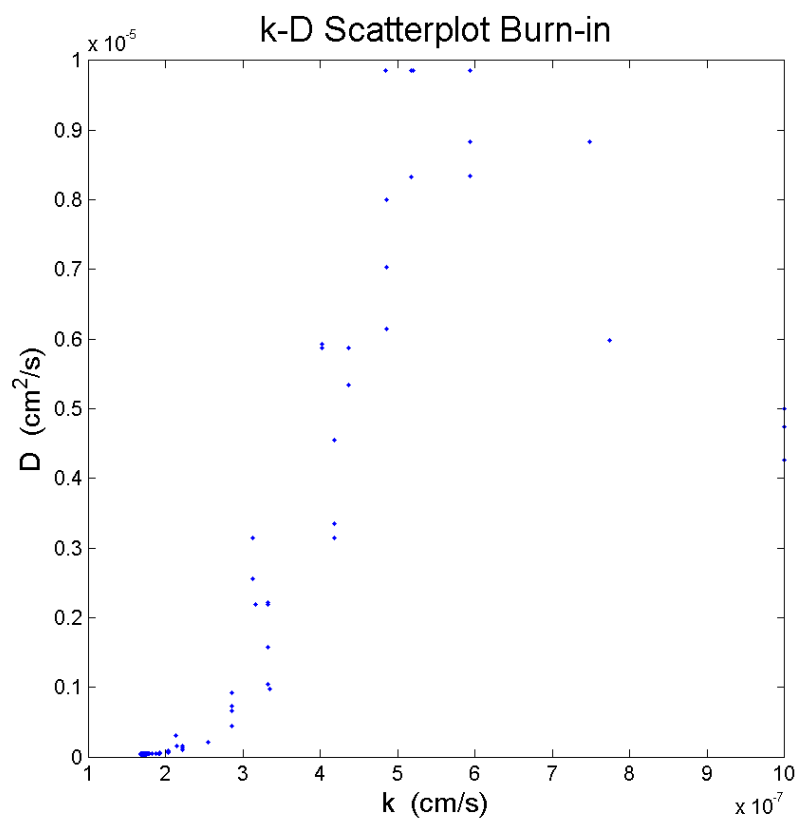

Figure 10: Scatter plot including the burn-in iterations for De-Souza's SIMS data.

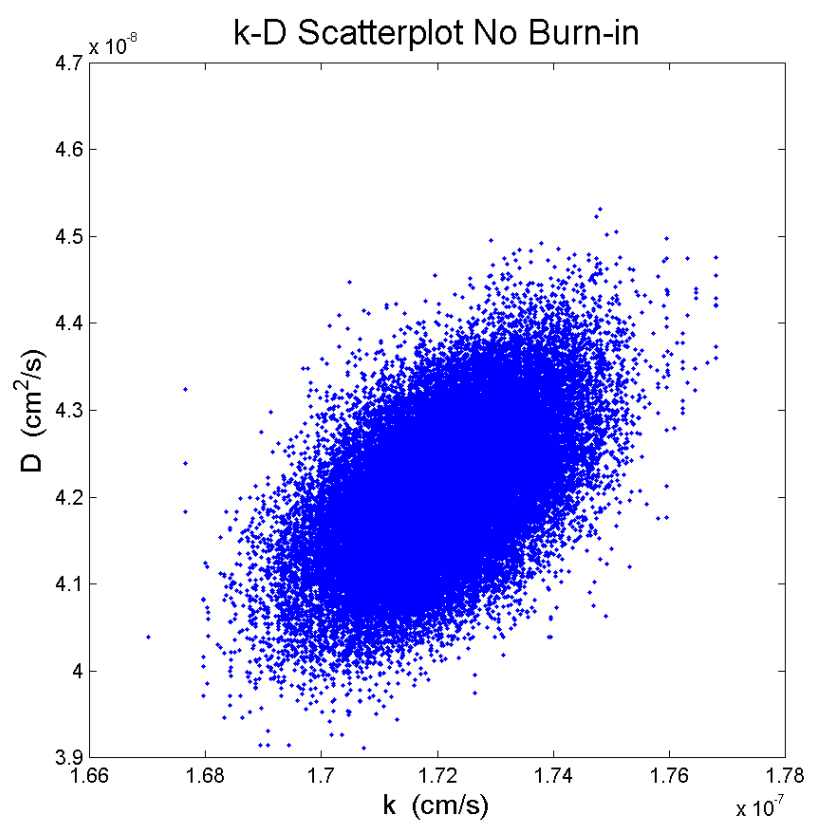

Figure 11: Scatter plot after removing burn in from 10.

clue as to how much more or less probable one parameter choice is over another within the span of the error bar. The results in table 1 and 2 agree nicely with the results given in the original paper. 


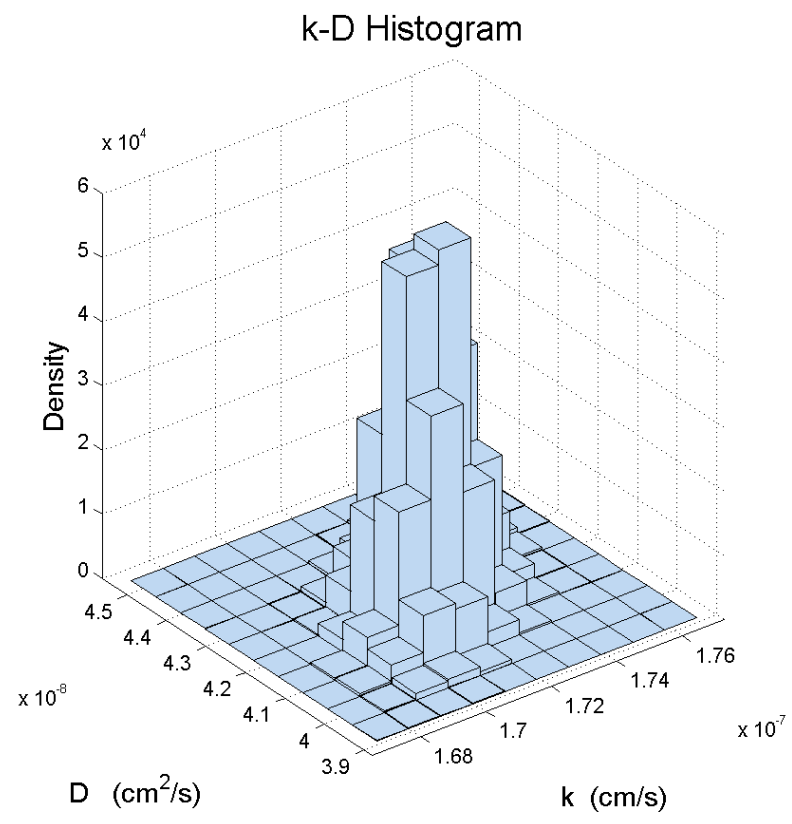

Figure 12: 3-D histogram showing the relative density in the various regions of the above scatter plot (fig. 11)

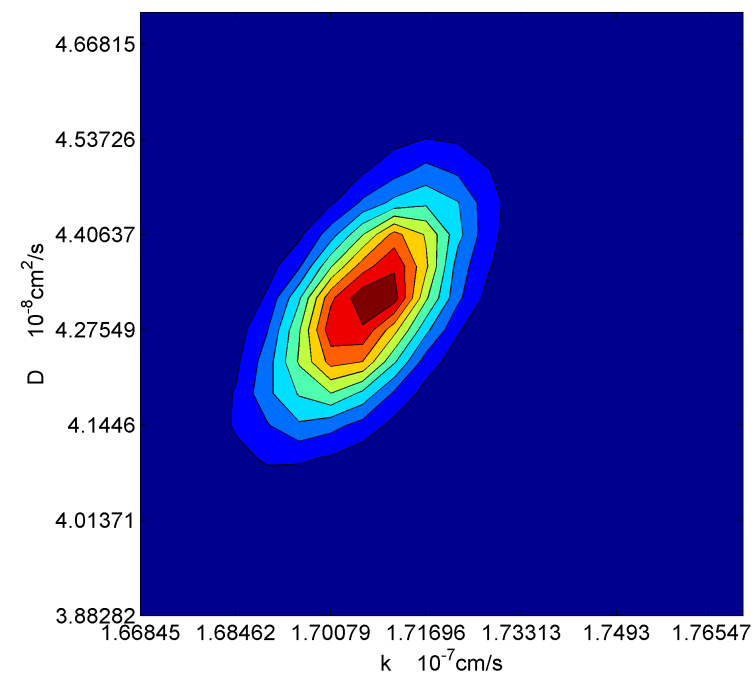

Figure 13: Contour plot produced directly from the 3-D histogram. Dark red regions correspond to highest density, while the blue corresponds to zero density.

Figure 14 shows the value of the likelihood with respect to iteration number. Within one iteration, the log-likelihood made a jump of over 1000, and within 50 iterations, the likelihood function had converged to its maximum region. Figure 15 is simply a zoomed-out version of figure 


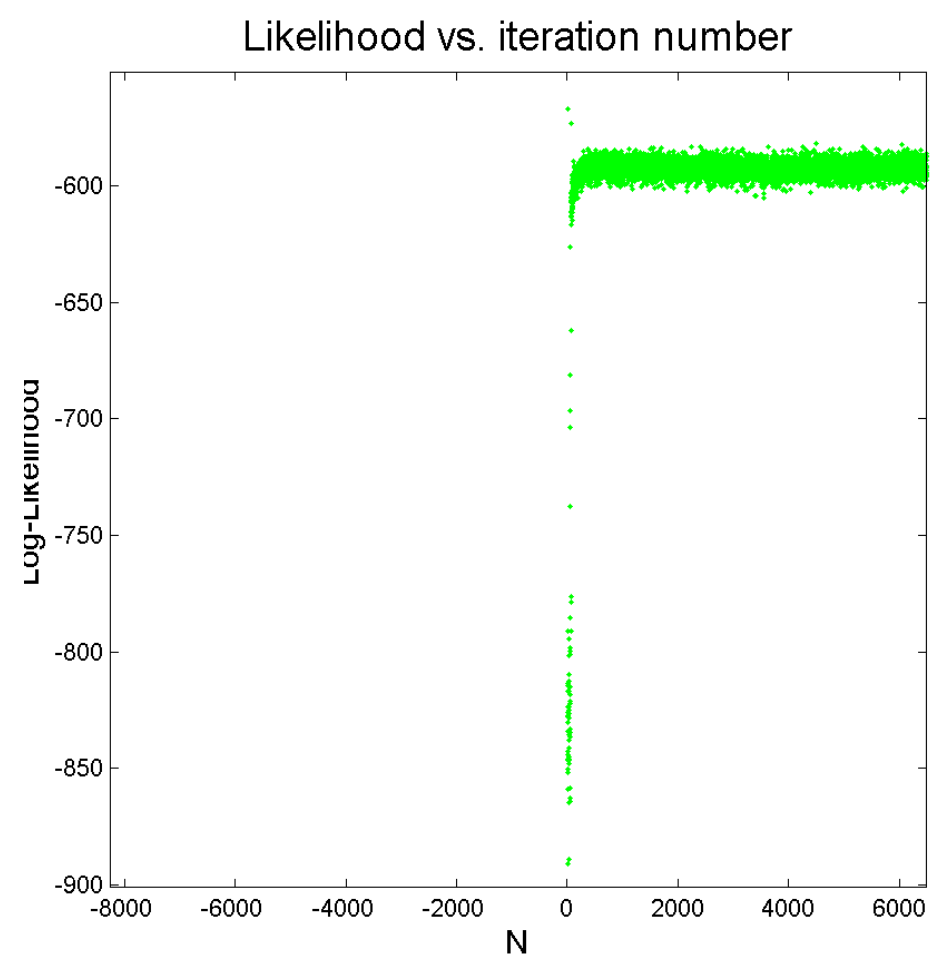

Figure 14: Value of the common log of the likelihood vs. iteration number, focused on the beginning iterations for SIMS data. Note that the heart of the distribution was found in less than 2000 iterations.

14, and shows the behavior of the likelihood for the entire simulation. Figures 16 and 17 are 1-D histograms of each parameter, for easy identification of the mode of the distribution. These figures are also useful for seeing how quickly the density decreases away from the mode, and therefore, how sensitive parameter changes are to the likelihood.

Figures 19 and 20 show how random draws from the posterior fit to the experimental data. The blue in figure 19 is actually a family of 2,000 curves. Figure 20 is a zoomed in view of figure 19, and more clearly shows the individual curves. It is seen from these figures that any draw from the posterior is a plausible fit. The posterior distributions of these studies do not include any values that produce unreasonable fits. This demonstrates the reliability of this technique and obtained results.

For the SIMS results, the routine finished in roughly five minutes for 500,000 samples on a Windows PC with an Intel Core I-7 3930k at $4.3 \mathrm{Ghz}$ with $32 \mathrm{~GB}$ of RAM on a 32-bit version of MATLAB. Results should be even faster on a 64-bit version since MATLAB would be able to utilize more than $4 \mathrm{~GB}$ of system RAM. 


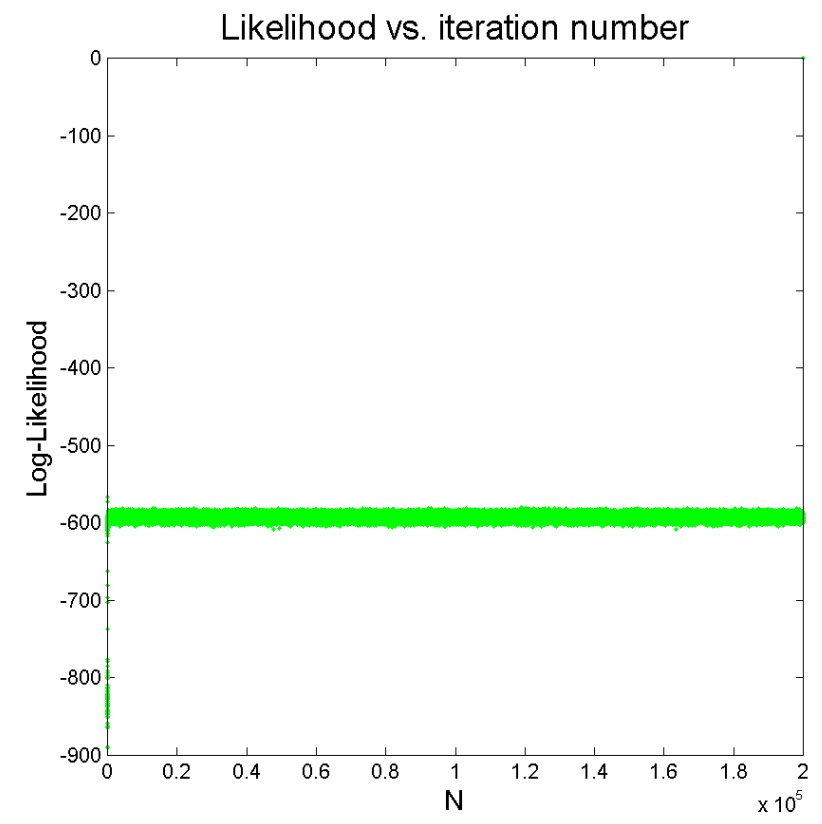

Figure 15: Global view of log-likelihood vs. iteration number for the entire routine.

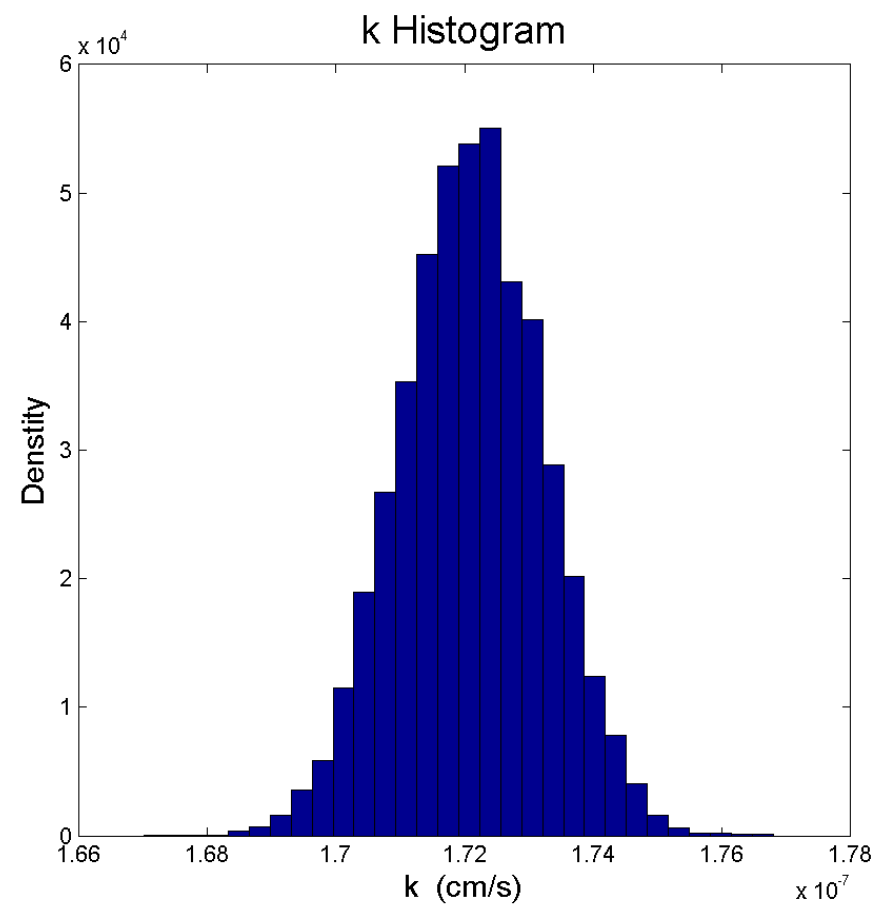

Figure 16: Histogram for the surface exchange coefficient of SIMS data. The height of the $i^{\text {th }}$ bar is the number of posteriors that fall between been the boundary of the bar throughout the routine. 


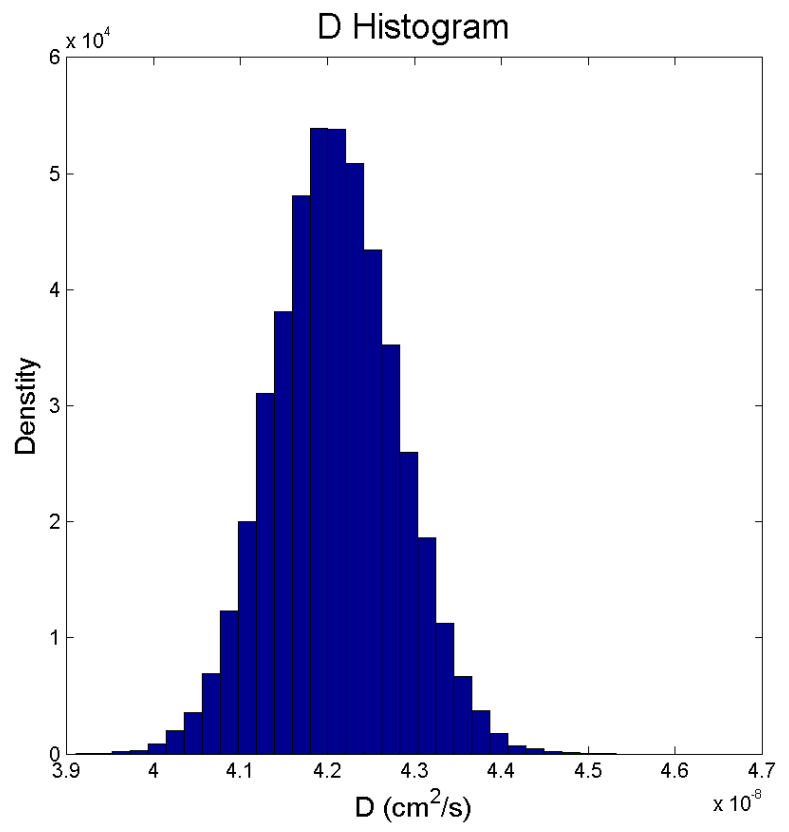

Figure 17: Histogram for the diffusion coefficient for SIMS data

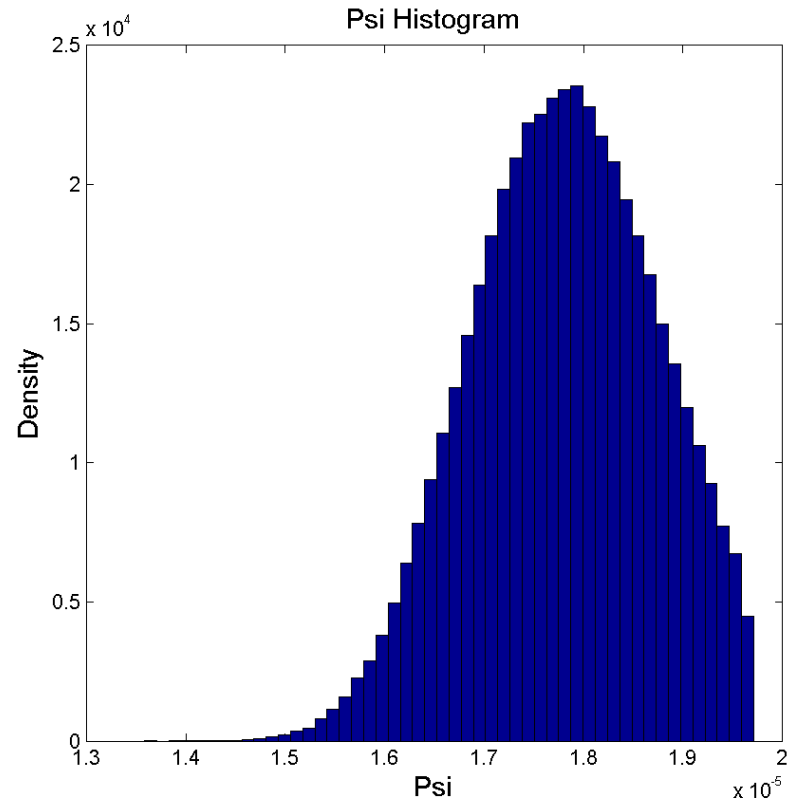

Figure 18: Psi histogram for SIMS data. 


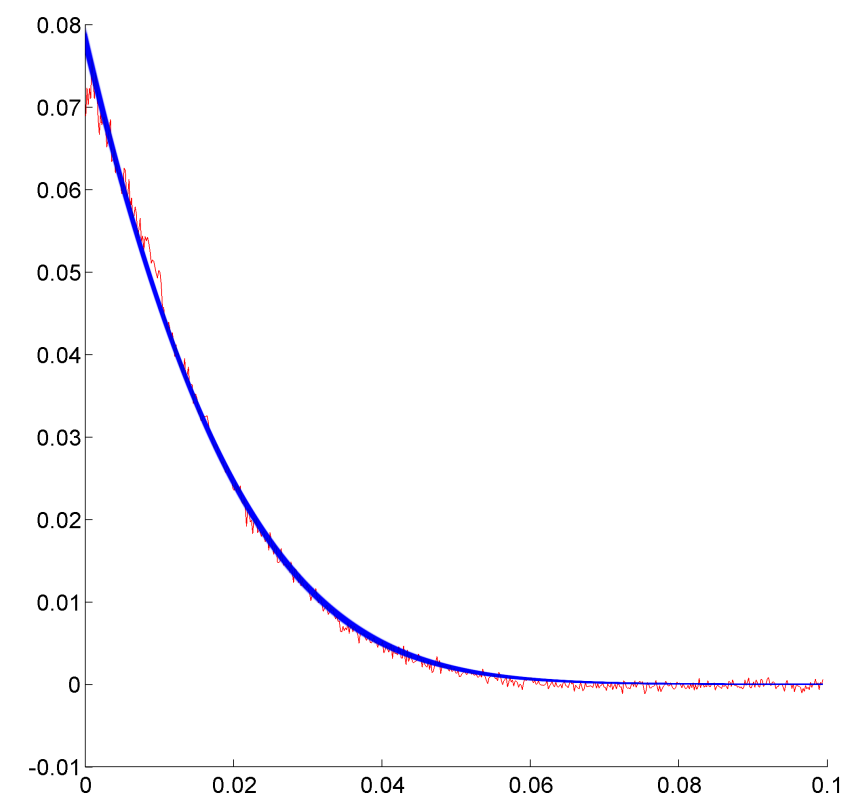

Figure 19: The blue portion is a family of 2,000 curves by drawing randomly from the posteriors. The red is the experimentally obtained data.

\subsection{Electrical Conductivity Relaxation}

In this study, data was pulled from two different papers[20, 21]. ECR experiments were conducted on $\operatorname{LSCF}(6428)$ at $1073 \mathrm{~K}$ at very similar partial pressures. The results for the surface exchange coefficient and bulk diffusion coefficient were widely varying between the two. This fact is an immediate example of a bigger problem. Many have conducted ECR experiments on the same materials in the same conditions and obtained different results for $\mathrm{k}$ and $\mathrm{D}$. The source of the discrepency is often put on the imprecision of ECR and is not investigated any further [11, 21]. However, these results show that the uncertainty associated with these particular ECR experiments, and probably other experiments as well, is not as high as other researchers have reported.

Table 3: 95 percent confidence intervals for the ECR analyses.

\begin{tabular}{|l|l|l|c|r|}
\hline Authors & $k_{\min }$ & $k \max$ & $D_{\min }$ & $D_{\max }$ \\
\hline McIntosh and Cox & $1.70 \times 10^{-3}$ & $2.60 \times 10^{-3}$ & $1.07 \times 10^{-5}$ & $1.33 \times 10^{-5}$ \\
\hline Lane and Kilner & $8.40 \times 10^{-5}$ & $10.6 \times 10^{-5}$ & $1.10 \times 10^{-6}$ & $1.24 \times 10^{-6}$ \\
\hline
\end{tabular}

Table 4: Modes for the ECR analyses.

\begin{tabular}{|l|l|l|}
\hline Authors & $k_{\text {mode }}$ & $D_{\text {mode }}$ \\
\hline McIntosh and Cox & $2.10 \times 10^{-3}$ & $1.18 \times 10^{-5}$ \\
\hline Lane and Kilner & $9.53 \times 10^{-5}$ & $1.15 \times 10^{-6}$ \\
\hline
\end{tabular}




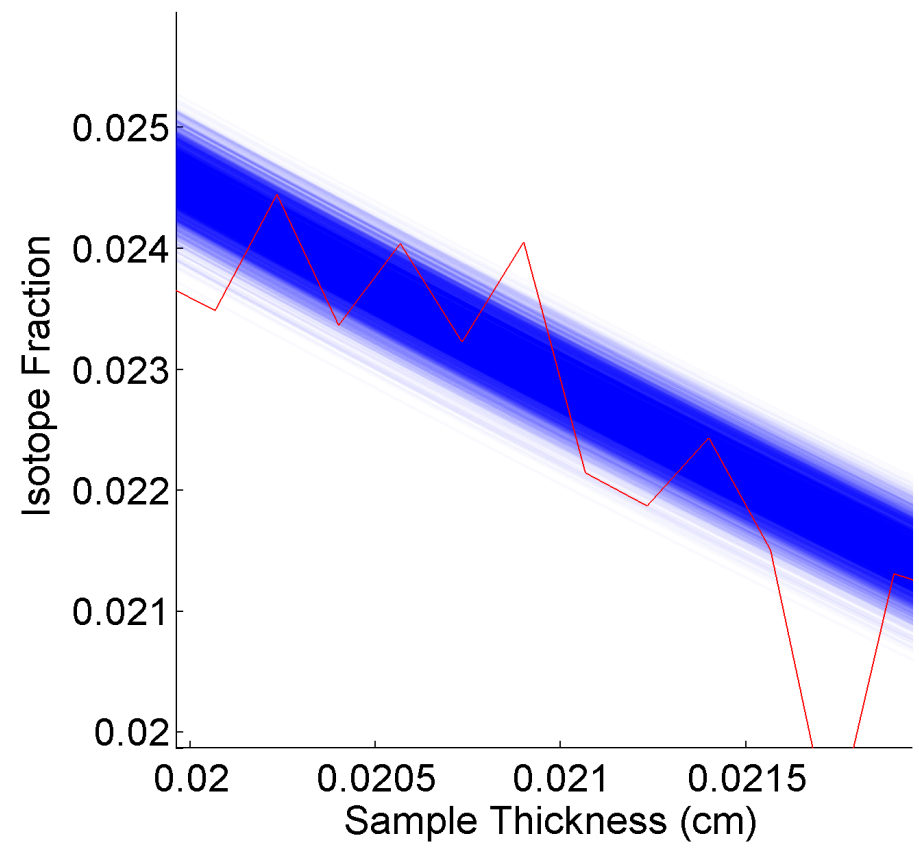

Figure 20: A zoomed in view of the graphic above that shows the relative width of the family of curves.

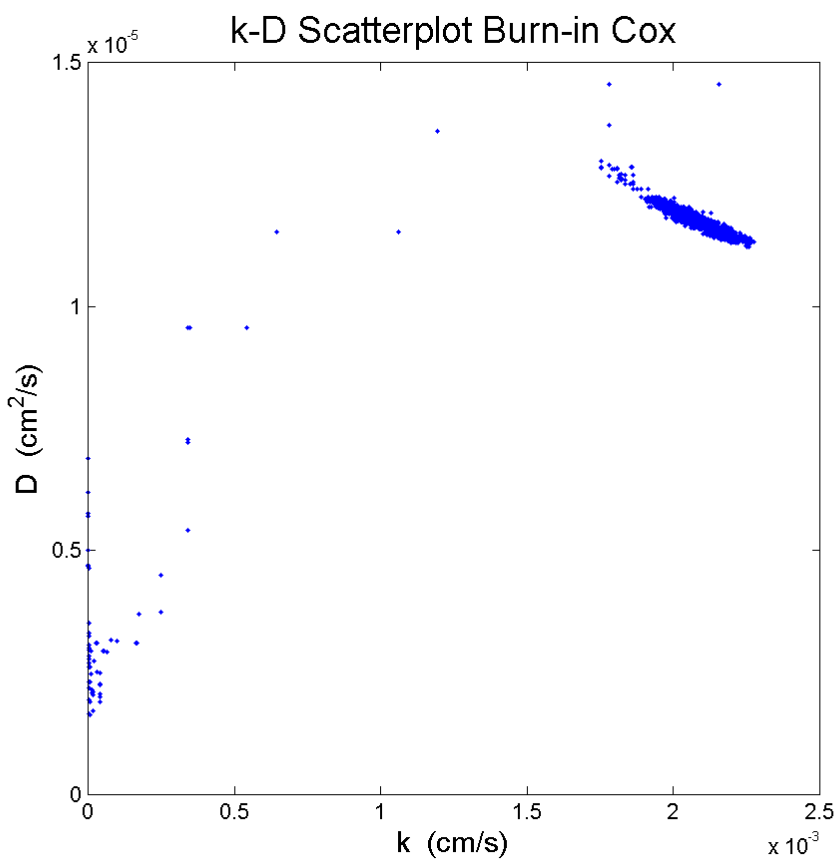

Figure 21: Brun in plot for the ECR data from the Cox and McIntosh paper. 


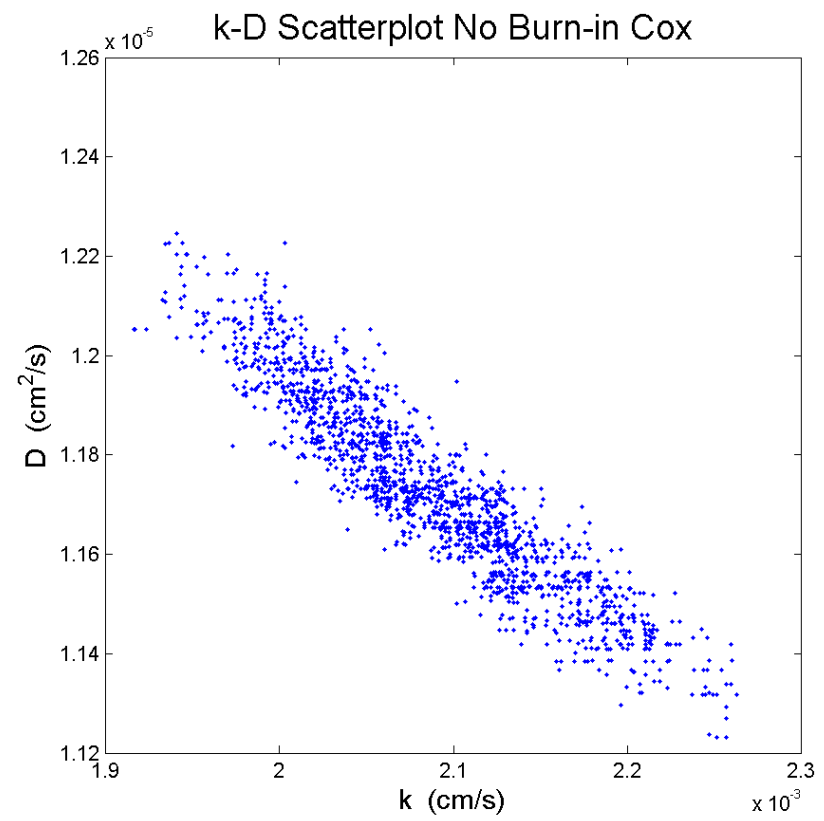

Figure 22: Scatter plot for the ECR data from the Cox and McIntosh paper.

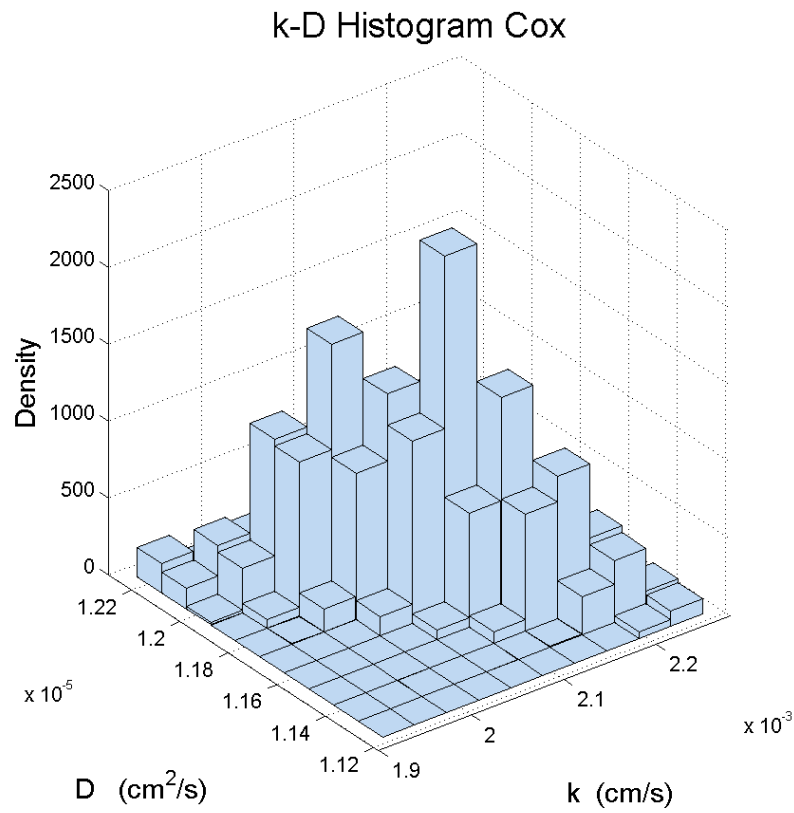

Figure 23: 3-D Histogram for the ECR data from the Cox and McIntosh paper. 


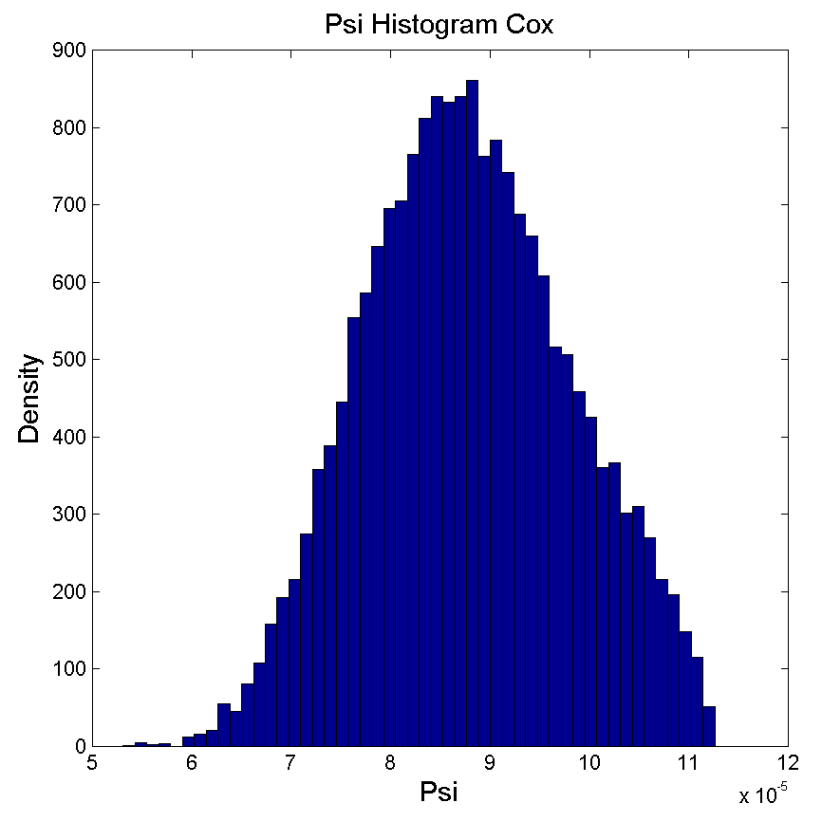

Figure 24: Psi histogram for the ECR data from the Cox and McIntosh paper.

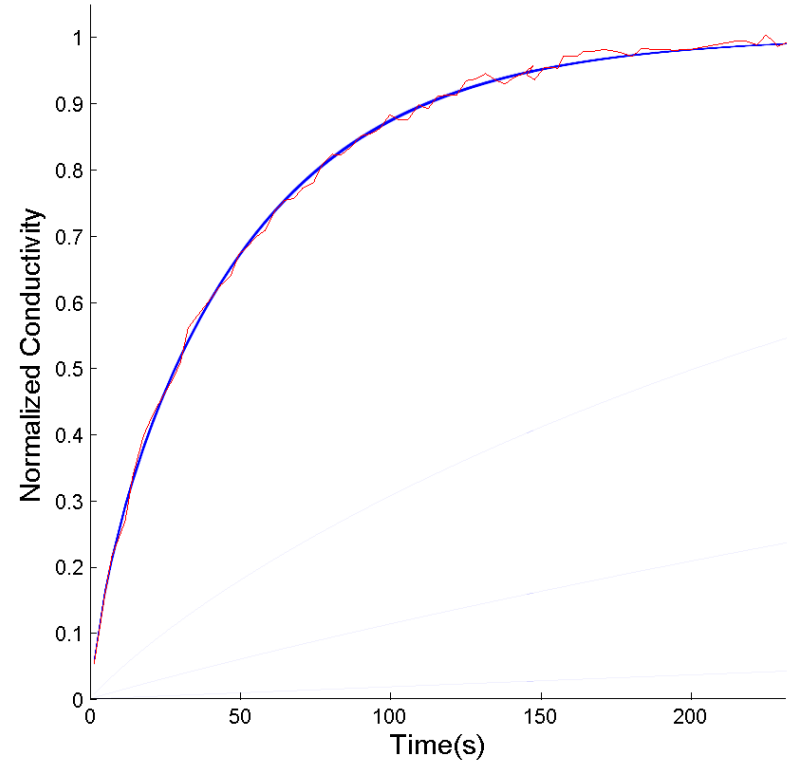

Figure 25: Predictions for the ECR data from the Cox and McIntosh paper. Burn in fits were included for both fit and thickness context. 


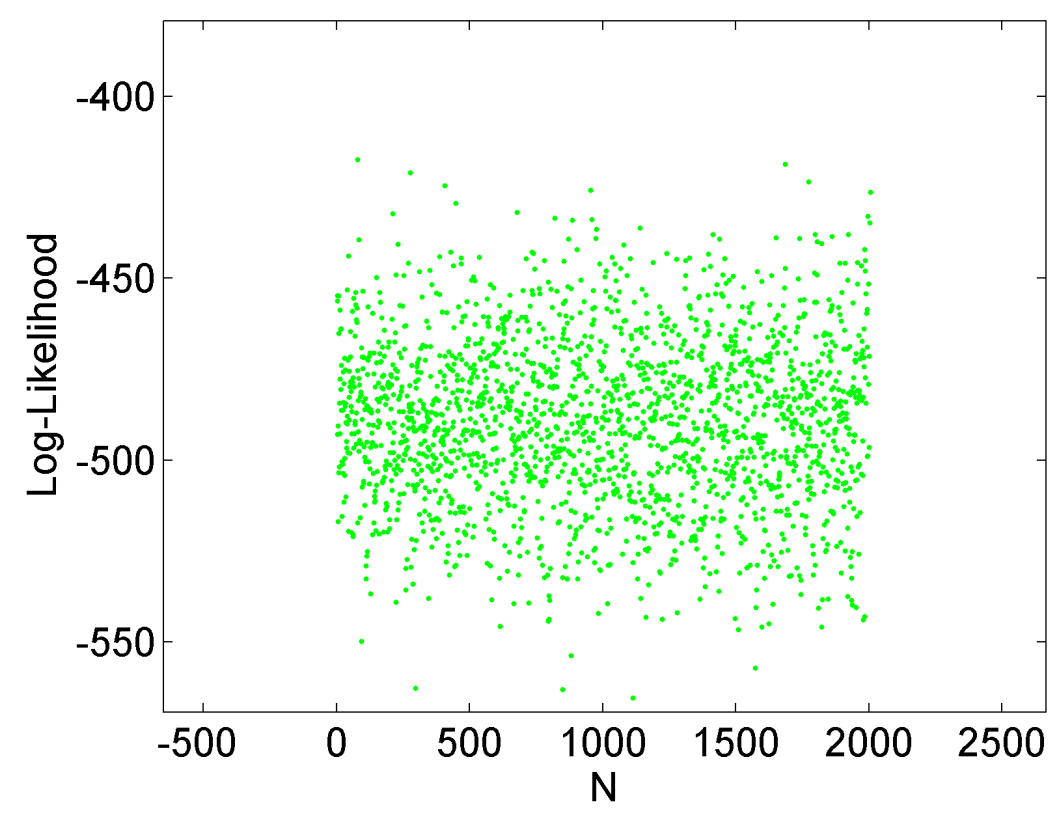

Figure 26: Zoomed in view of the first 2000 iterations of the log-likelihood. Note that these are burn-in samples, and are "stuck" at a maximum between -450 and -400 .

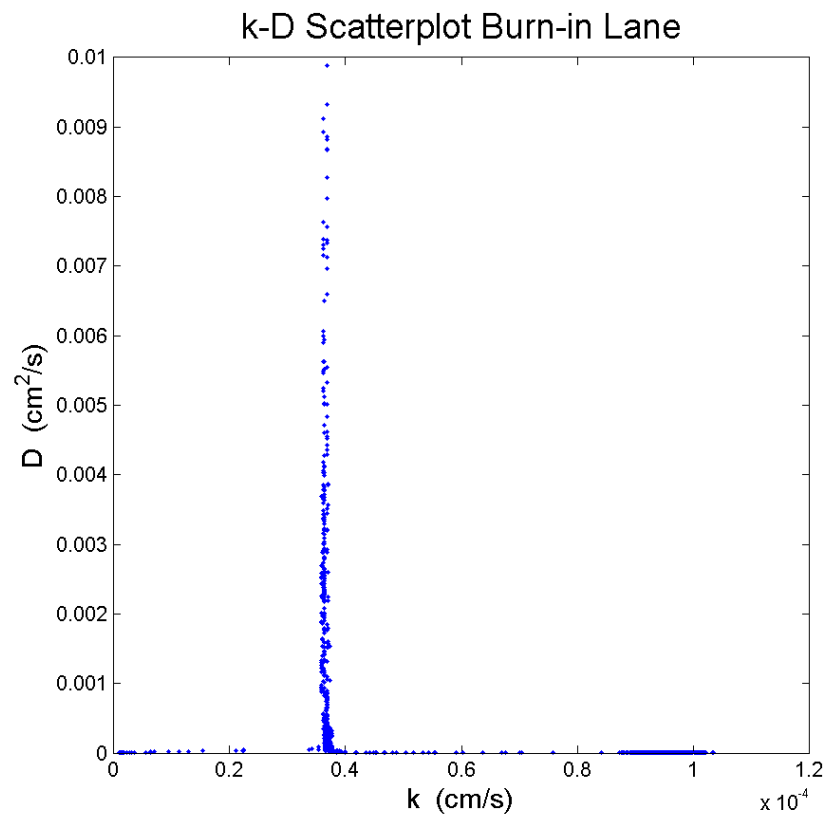

Figure 27: Burn in plot for the ECR data from the Lane and Kilner paper. 


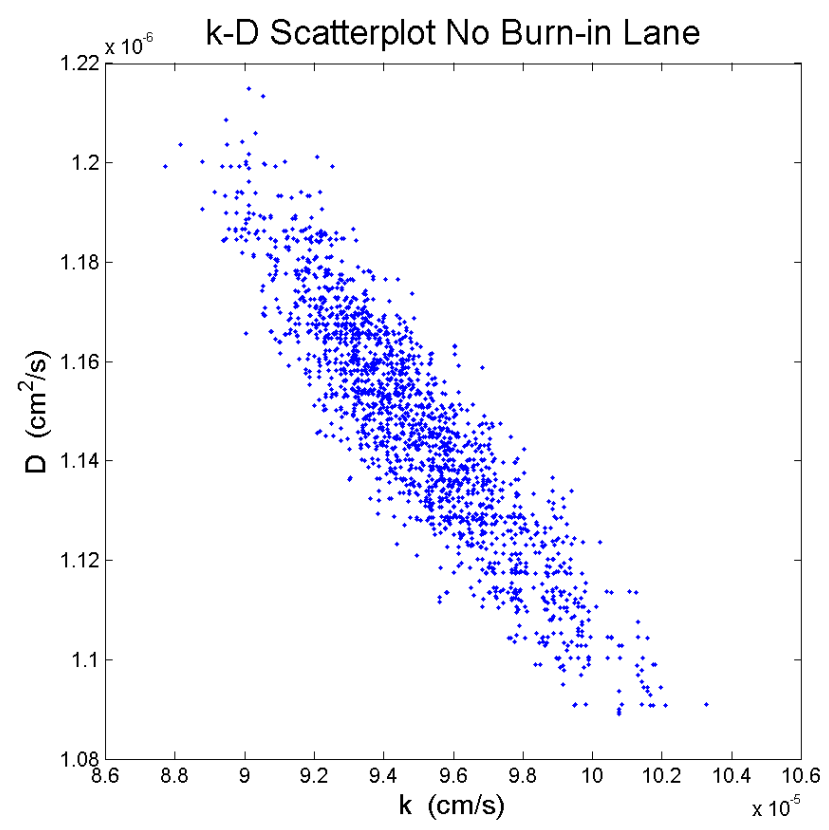

Figure 28: Scatter plot for the ECR data from the Lane and Kilner paper. Notice the relative elongation along the k-axis, showing a higher degree of unidentifiability.

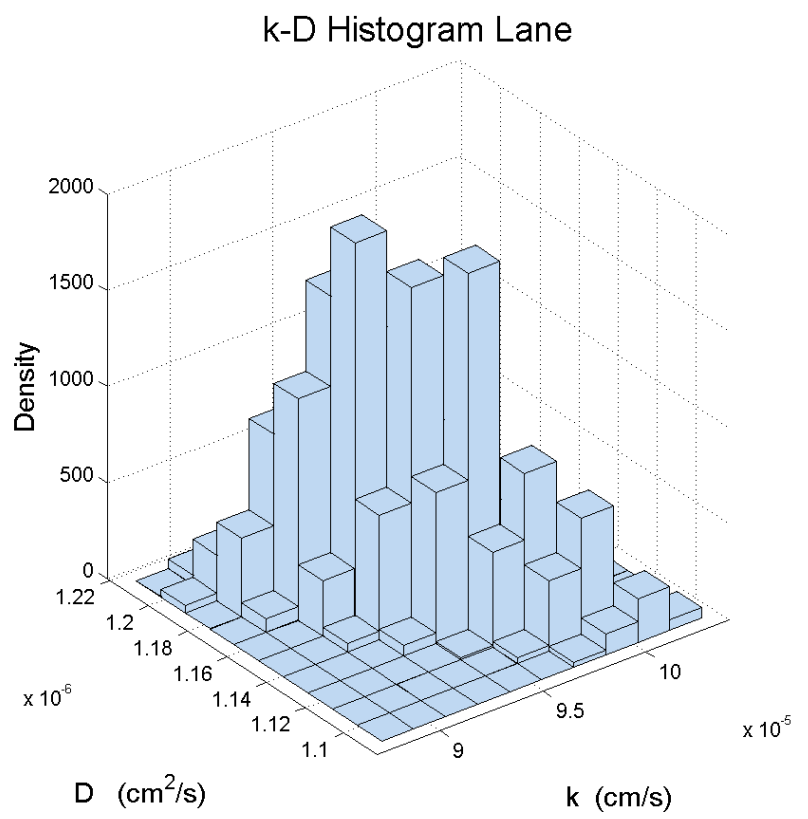

Figure 29: 3D histogram for the ECR data from the Lane and Kilner paper. 


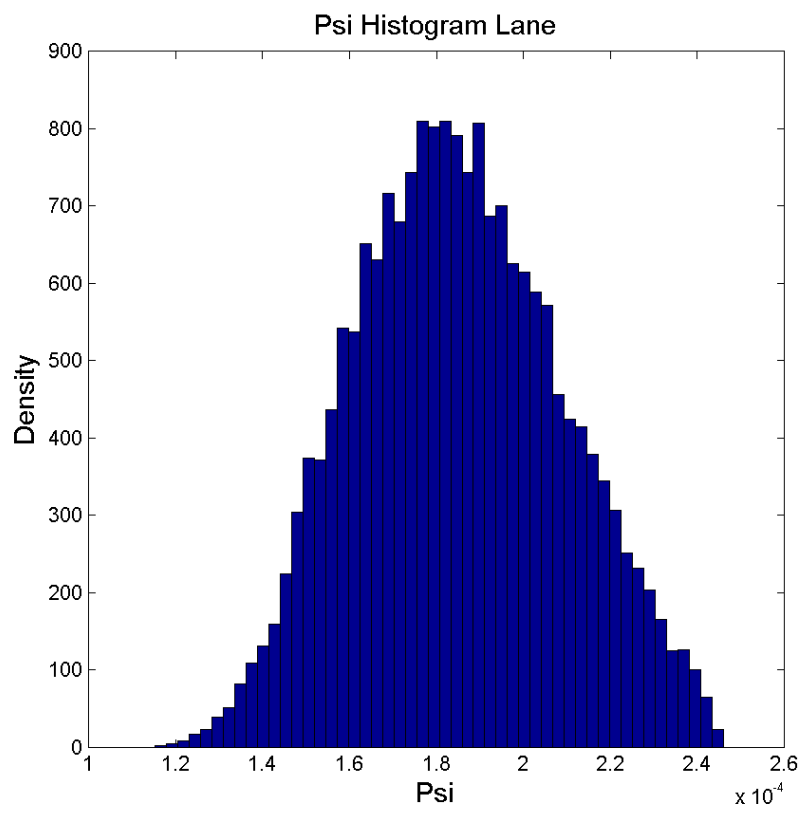

Figure 30: Psi histogram for the ECR data from the Lane and Kilner paper. 


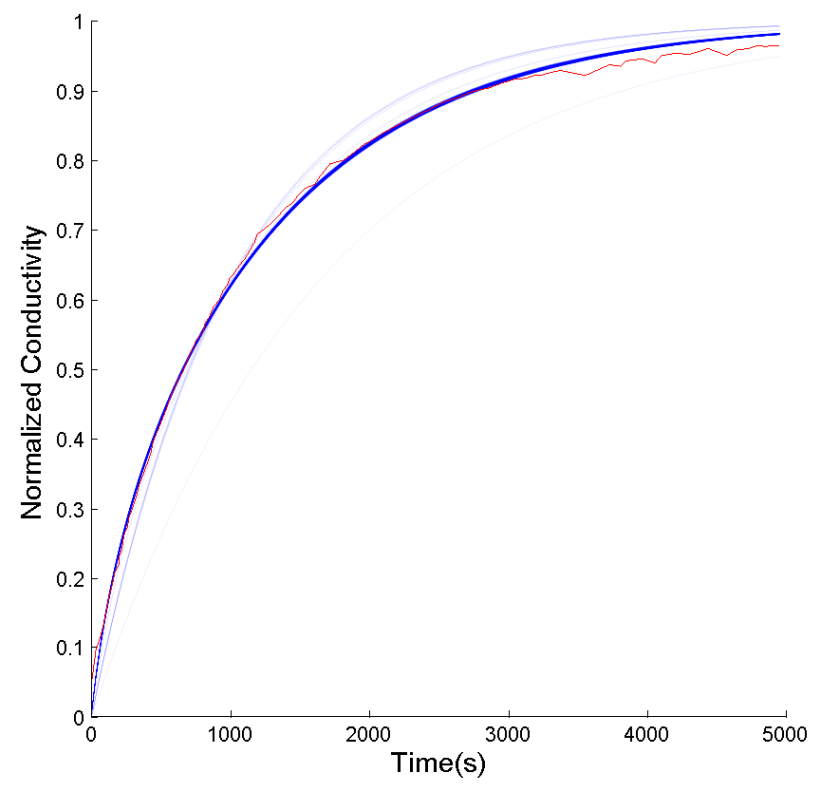

Figure 31: Predictions for the ECR data from the Lane and Kilner paper.

Figures 21 through 25 correspond to the results from analyzing the data from Cox and McIntosh, while figures 26 through 30 correspond to the data pulled from Lane and Kilner's paper. If the uncertainty was as high as others have stated, then there would be a clear region where the distributions obtained from analyzing the datasets from each paper would overlap. That is, the uncertainty in $\mathrm{k}$ and $\mathrm{D}$ from paper 1 would be such that the result from paper 2 would be contained within the error bars in paper 1. However, upon analyzing the data from each paper, it is observed that the distributions from each dataset are mutually exclusive. In particular, by examining figures 23 and 29 , the 3D histograms from each data set, it is obvious that the two distributions have no intersection.

The meaning of all of the input parameters of the routine will now be discussed. $k_{\min }, k_{\max }$, $D_{\min }$, and $D_{\max }$ are the bounds for the uniform priors on $\mathrm{k}$ and $\mathrm{D}$. No value outside the interval specified will be considered at all. "N" is the number of posterior samples obtained before writing the results to the hard drive. This allows one to analyze the progression of posteriors while the routine is running. This is particularly useful when the function being used to model the data is computationally cumbersome. "BT" is the number of files that will be written to the hard drive during the course of the routine, with each file having " $\mathrm{N}$ " samples. "P" is the type of confidence interval that is to be calculated. For example, setting $\mathrm{P}=95$ will calculate the 95 percent confidence intervals for $\mathrm{k}, \mathrm{D}$, and psi. $k_{95}, D_{95}$, and $\psi_{95}$ is the vector corresponding to the numbers calculated from choosing "P". " $N_{c}$ " is the number of curves used in the "patchline" plot. This is, the number of curves produced by taking $N_{c}$ posterior samples and producing a fit curve with each sample. Refer to figures (25) and (31) for an illustration. $D_{b i n s}, k_{b i n s}$, and $\psi_{\text {bins }}$ are refer to the resolution of the $\mathrm{D}, \mathrm{k}$, and psi histograms, respectively. $X_{\text {bins }}$ and $Y_{\text {bins }}$ refer to the resolution of the 3-D histogram and contour plot, as in figure (12) and (13). 


\section{Conclusions}

By producing a set of idealized data, it has been shown without a doubt that this technique is capable of quickly locating the "true" neighborhood of parameters. In all studied cases thus far, parameter modes are located in less than 5,000 iterations. Although a completely fool-proof method for ensuring the routine is not stuck at a local max has not been developed, the graphics included in this routine make it very easy for one to inspect the unexplored regions of parameter space. Setting $\varsigma$ to be small enough will ensure that a maximum is not skipped when searching in unexplored regions. The behavior of the routine on ideal data, along with the consistent results that were obtained when applying it to real SIMS data, prove this technique to be simple, accurate, precise, and computationally advantageous over other more traditional methods. When applying the simulation to two sets of ECR data on the same material under nearly identical experimental conditions, two different distributions with no intersection were obtained. Due to this fact, it can be safely concluded that the uncertainty pertaining to ECR is lower than others have previously reported, and these findings also support the notion that ECR is still a reliable and relatively inexpensive and easy way to obtain material transport properties, even though IE-SIMS is still the top contender of such experiments. 


\section{References}

[1] I. Riess, Mixed Ionic-electronic conductors -material properties and applications Solid State Ionics Volumne 157, Issues 1-4, Pages 1-17, February 2003.

[2] A. Mitterdorfer, L.J. Gauckler, Identification of the reaction mechanism of the Pt, $O_{2}(g)$-yttirastabilized zirconia system-PartI: General framework, modeling, and structural investigation, J. Solid State Ionics 117 (3-4) (1999) 187-202.

[3] D. S. Tannhauser, J. A. Kilner, B. C. H. Steele, The determination of the oxygen self-diffusion and gas solid exchange coefficients for stabilized zirconia by SIMS, Nucl. Inst. Meth. Phys. Res. 218 (1-3) (1983) 504-508.

[4] J. A. Kilner, B. C. H. Steele, L. Ilkov, Oxygen self diffusion studies using 16negative-ion secondary ion mass spectrometry (sims), Solid State Ionics 24512 (MAR) (1984) 89-97.

[5] J. M. Bassat, M. Petitjean, J. Fouletier, C. Lalanne, G. Caboche, F. Mauvy, J. C. Grenier, Oxygen isotopic exchange: A useful tool for characterizing oxygen conducting oxides, Appl. Catal. A 289 (1) (2005) 84-89.

[6] I. Yasuda, T. Hikita, Precise determination of the chemical diffusion- 250 coefficient of calciumdoped lanthanum chromites by means of electricalconductivity relaxation, J. Electrochem. Soc. 141 (5) (1994) 1268-1273.

[7] I. Yasuda, M. Hishinuma, Electrical conductivity and chemical diffusion coefficient of strontium-doped lanthanum manganites, J. Solid State Electrochem. 123 (2) (1996) 382-390.

[8] Ronald K. Wangsness, Electromagnetic Fields, 2nd Edition, John Wiley and Sons, 1986, page $68,208$.

[9] W. G. Bessler, A new computational approach for SOFC impedance 260 from detailed electrochemical reaction-diffusion models, Solid State Ionics 176 (11-12) (2005) 997-1011.

[10] D. S. Mebane, Y. Liu, M. L. Liu, Refinement of the bulk defect model for $\mathrm{La}_{x} \mathrm{Sr}_{1 x} \mathrm{MnO}_{3}$, Solid State Ionics 178 (2008) 1950-1957

[11] Y. H. Li, K. Gerdes, H. Diamond, X. B. Liu, An improved method to increase the predictive accuracy of the ECR technique, Solid State Ionics 204 (2011) 104-110.

[12] A. Gelman, J. B. Carlin, H. S. Stern, D. B. Rubin, Bayesian Data Analysis, CRC Press, 2004.

[13] D. Mebane, K. Bhat, J. Kress, D. Fauth, M. Gray, A. Lee, D. Miller, Bayesian calibration of thermodynamic models for the uptake of CO2 in 18supported amine sorbents using ab initio priors, Phys. Chem. Chem. Phys. 30015 (2013) 4355-4366.

[14] G. L. Jones, M. Haran, B. S. Caffo, R. Neath, Fixed-width output analysis for Markov chain Monte Carlo, J. Am. Stat. Assoc. 101 (476) (2006) 1537- 1547.

[15] Andrew Gelman, Objections to Bayesian Statistics, International Sociaety for Bayesian Analysis, Bayesian Analysis, (2008) pp. 445-450 
[16] J. Crank, The Mathematics of Diffusion, 2nd Edition, Oxford University Press, 1975.

[17] A. Gelman, D. B. Rubin, Inference from iterative simulation using multiple sequences, Stat. Sci. 7 (4) (1992) 457-472.

[18] G. L. Jones, M. Haran, B. S. Caffo, R. Neath, Fixed-width output analysis for Markov chain Monte Carlo, J. Am. Stat. Assoc. 101 (476) (2006) 1537- 1547.

[19] M. J. Pietrowski, R. A. De Souza, M. Fartmann, R. ter Veen, M. Martin, Oxygen isotope transport properties of yttria-stabilized zirconia (YSZ) in $\mathrm{O}_{2}$ and $\mathrm{H}_{2} \mathrm{O}$-containing atmospheres, Fuel Cells 13 (5) (2013) 673-681.

[20] J. A. Lane, J. A. Kilner, Measuring oxygen diffusion and oxygen surface exchange by conductivity relaxation, Solid State Ionics 136 (2000) 997- 3151001.

[21] R. A. Cox-Galhotra, S. McIntosh, Unreliability of simultaneously determining $k_{\text {chem }}$ and $D_{\text {chem }}$ via conductivity relaxation for surface-modified $\mathrm{La}_{0.6} \mathrm{Sr}_{0.4} \mathrm{Co}_{0.2} \mathrm{Fe}_{0.8} \mathrm{O}_{3}$, Solid State Ionics 181 (31-32) (2010) 1429-1436. 
Student Name: $\frac{\text { Bia } a^{\prime} r}{\text { (Last) }}$

Student ID\#: 700939347
Joshua

(First)
Lee

(Middle)
Degree:

Document Type:

Document Title:
Non-WVU Email Account: Jblair@mail Kana,kiz, wv, us

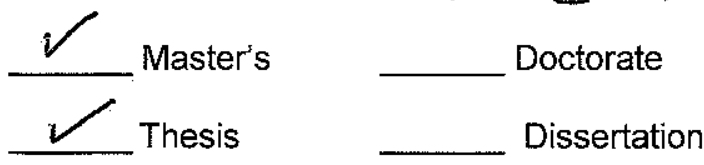

A Bayesian Approach to Uncertainty Quantification of Electrical conductivity Relaxafion and Ksotope Exchande / Serondany Ion mass

\section{Student Agreement:}

I hereby certify that, if appropriate, I have obtained and attached hereto a written permission statement from the owners of each third party copyrighted matter to be included in my thesis, dissertation, project report, or other research material, allowing distribution as specified upon deposit.

I hereby grant to West Virginia University and its agents the non-exclusive license to archive and make accessible, under the conditions selected upon deposit, my above mentioned document in whole or in part in all forms of media, now or hereafter known. I retain ownership rights as specified in the WVU copyright policy to the copyright of the abovementioned document. I also retain the right to use in future works (such as articles or books) all or part of this abovementioned document.

\section{Review and Acceptance:}

The above mentioned document has been reviewed and accepted by the student's advisory committee. The undersigned agree to abide by the statements above, and agree that this Signature Form updates any and all previous Signature Forms submitted heretofore.

Signed:

Committee:
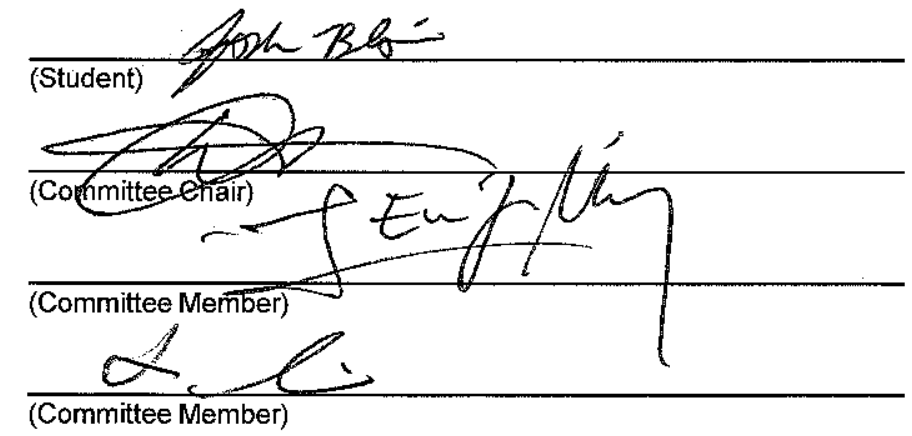

(Committee Member)

(Committee Member)

(Committee Member)

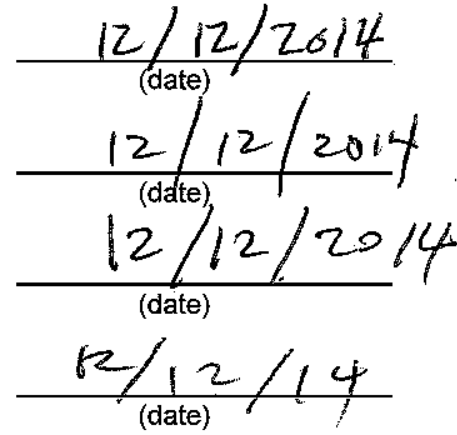

(date)

(date)

(date) 